\title{
Recent developments and trends in the application of strontium and its isotopes in biological related fields
}

$\underline{\text { Inês Coelho }}{ }^{\mathrm{a}}$, Isabel Castanheira ${ }^{\mathrm{a}}$, João Moura Bordado ${ }^{\mathrm{b}}$, Olivier Donardc ${ }^{*}$, José Armando L. Silva ${ }^{\mathrm{d}^{*}}$

*Corresponding author: olivier.donard@univ-pau.fr

* Corresponding author: pcd1950@tecnico.ulisboa.pt

a Departamento de Alimentação e Nutrição, Instituto Nacional de Saúde Doutor Ricardo Jorge, INSA. IP, Avenida Padre Cruz, 1649-016 Lisboa, Portugal. ines.coelho@insa.min-saude.pt, isabel.castanheira@insa.min-saude.pt ${ }^{b}$ CERENA, Departamento de Engenharia Química e Biológica, Torre Sul, Instituto Superior Técnico, Universidade de Lisboa, Av. Rovisco Pais, 1049-001 Lisboa, Portugal. jcbordado@ist.utl.pt

${ }^{\circ}$ Center of Mass Spectrometry for Reactivity and Speciation Sciences, IPREM UMR 5254, Université de Pau et des Pays de l'Adour, Hélioparc, 64053 Pau, France. olivier.donard@univ-pau.fr d Centro de Química Estrutural, Instituto Superior Técnico, Universidade de Lisboa, Av. Rovisco Pais, 1, 1049-001 Lisboa, Portugal. pcd1950@tecnico.ulisboa.pt

\section{Contents}

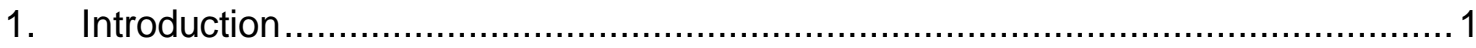

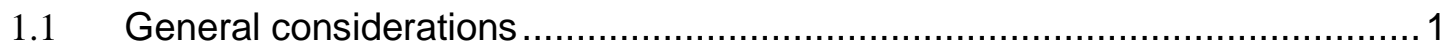

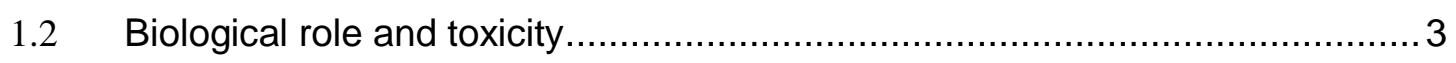

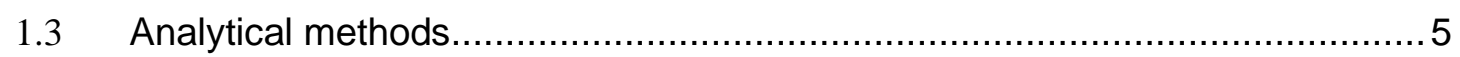

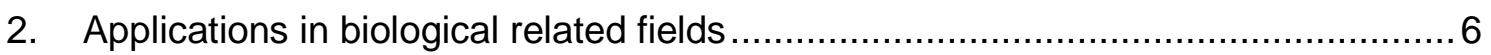

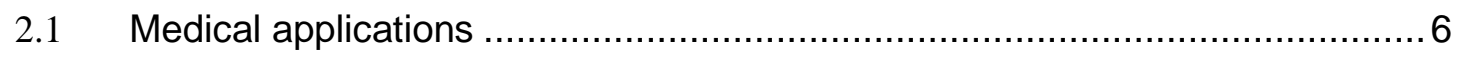

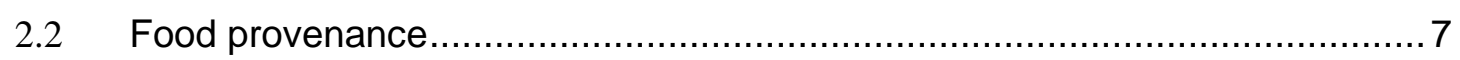

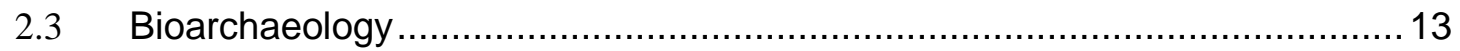

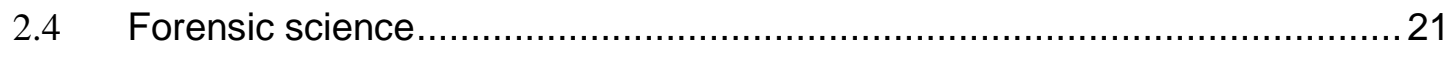

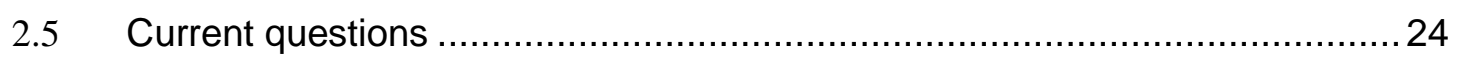

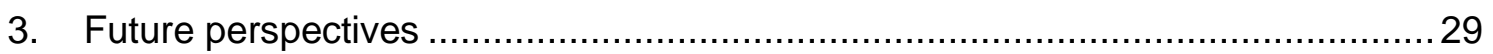

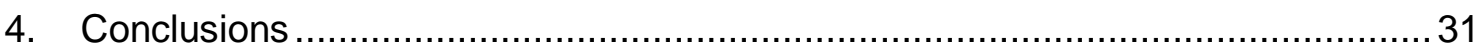

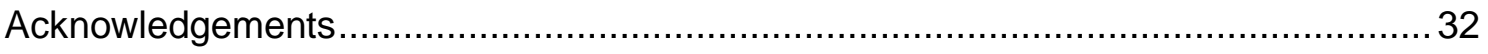

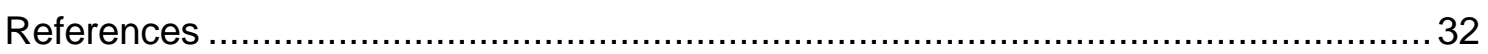




\section{Abstract}

3 Strontium is a chemical element without biological functions in humans. In fact, only

4 two biological roles are known, both as a biomineral constituent. However, strontium

5 and calcium (a significant biometal) present chemical similarities; hence, the absorption

6 of the former by living organisms is plausible. Based on the particular properties of

7 isotopes of strontium several applications of this chemical element in biological

8 sciences have been developed. These include its radioactive and non-radioactive

9 isotopes in medical applications or resorting to its stable isotopes as analytical tools in

10 isotope fingerprinting $\left({ }^{87} \mathrm{Sr} /{ }^{86} \mathrm{Sr}\right)$ to study dietary habits and migration patterns of

11 ancient populations, food authentication and forensic sciences. This article, reviews

12 papers published between 2012-2016, highlights the use of strontium isotope ratios for

13 such biological related applications and compiles an analytical database, providing a

14 valuable tool for future studies in the area.

\section{Keywords}

17 Strontium; isotope ratio; biological marker; medicine; food authentication; dietary

18 habits; migration pattern; forensic science; MC ICP-MS; TIMS

\section{List of abbreviations}

21 A - amplitude of the interval

ATSDR - Agency for Toxic Substances and Disease Registry

$\mathrm{BC}$ - before christ

bw - bodyweight

ca - circa, approximately

CHILI - chicago instrument for laser ionization

27 ICP-Q-MS - inductively coupled plasma quadrupole mass spectrometry

28 IRMS - isotope ratio mass spectrometry

n.a. - not available

34 RSD - relative standard deviation

35 TIMS - thermal ionization mass spectrometry

36 TPB - total procedural blank 


\section{Introduction}

\subsection{General considerations}

Strontium is a very reactive metal that quickly oxidizes forming an oxide and therefore it does not occur free in nature [1]. It is a lithophile metallic element, which is found in nature as $\mathrm{Sr}^{2+}$, and may substitute $\mathrm{Ca}^{2+}$ in a variety of rock-forming minerals, particularly Ca rich minerals, including feldspar, gypsum, plagioclase, apatite and, especially, calcite and dolomite [2,3]. Sr in rocks is found with variable concentrations depending upon their nature and composition. In igneous rocks $\mathrm{Sr}$ is enriched in basalts, andesites and dacites, while insignificant in ultramafic rocks [4,5]. In sedimentary rocks $\mathrm{Sr}$ is enriched in carbonate rocks [5].Weathering is responsible for releasing strontium from rocks into soils and subsequently to plants and animals, and for entering the oceans primarily by river transport of sediments [6]. Human activities also contribute to the release of strontium into the atmosphere, as aerosols [7]. Strontium has four stable isotopes ${ }^{88} \mathrm{Sr},{ }^{87} \mathrm{Sr},{ }^{86} \mathrm{Sr}$ and ${ }^{84} \mathrm{Sr}$ with relative average Earth's abundances of $82.5845 \%, 7.0015 \%, 9.8566 \%$ and $0.5574 \%$, respectively [8]. These were formed during the stellar synthesis [7]. ${ }^{87} \mathrm{Sr}$ has the particularity of being the only stable isotope of strontium that is radiogenic. The radioactive decay of ${ }^{87} \mathrm{Rb}$ (half-life $\approx$ $9.23 \times 10^{9}$ years [9]) into the radiogenic isotope ${ }^{87} \mathrm{Sr}$ leads to an increase in the natural relative abundance of the last, which is more significant for both higher concentrations of $\mathrm{Rb}$ and age of the soils as shown in Fig. $1 .{ }^{87} \mathrm{Sr} /{ }^{86} \mathrm{Sr}$ is considered a valuable tracer of strontium in rocks and minerals since it can be related to their $\mathrm{Rb} / \mathrm{Sr}$ [10], providing valuable information on both age and geochemical origin of the geologic materials $[5,11]$. Current ${ }^{87} \mathrm{Sr} /{ }^{86} \mathrm{Sr}$ values of geochemical reservoirs and rocks can be found between 0.702 for the depleted mantle (low $\mathrm{Rb} / \mathrm{Sr}$ ) and above 0.943 for old continental crust [12]

Once absorbed by plants the ${ }^{87} \mathrm{Sr} /{ }^{86} \mathrm{Sr}$ value is kept across the trophic levels since, unlike the organic components of foodstuffs that can be influenced by agronomical conditions [13] or by technological factors (e.g. extraction systems, processing filtrations, storage conditions) [14,15], Therefore, ${ }^{87} \mathrm{Sr} /{ }^{86} \mathrm{Sr}$ is constant across the food web, since they are not apparently influenced by external factors (e.g. pollution, climate) or fractionation after absorption by living organisms $[5,6]$. Such has been demonstrated by matching fish scales and otoliths to the surrounding water [16] or by studying strontium isotope ratios across three trophic levels (soil-plant-butterfly) [17]. In 
all organisms), hence promoting the uptake of the former by plants and animals [6], regardless of its biological relevancy. Therefore ${ }^{87} \mathrm{Sr} /{ }^{86} \mathrm{Sr}$ in biological samples, independently of the trophic level, can be used as tracers for geographical provenance since they reflect the original sources of strontium. Also radiogenic isotopes can be used as geochemical tracers contributing in studies of chemical weathering and soil genesis, cation provenance and mobility, and the chronostratigraphic correlation of marine sediments[5]. Isotopic systems have been widely employed in various fields of research and several applications, including in the Health Sciences [18]. It is possible to find several works since the 1970s dealing with isotope geochemistry [19], climate and environmental concerns $[20,21]$ or even archaeology [22]. If at first, the most common isotopes in such researches were mainly from light elements, such as hydrogen, oxygen, carbon, nitrogen and sulphur; developments in the analytical techniques have allowed broadening the range of elements, including the isotopic analysis of heavier elements such as strontium.

Additionally to its four stable isotopes, strontium has twenty nine radioactive isotopes [7], which defines as the property of a nuclide of undergoing spontaneous nuclear transformations with the emission of radiation [23], The unstable ${ }^{89} \mathrm{Sr}$ and ${ }^{90} \mathrm{Sr}$ result from anthropogenic activity [24]. They are formed during nuclear reactor operations and nuclear explosions by the fission of uranium and plutonium $\left({ }^{235} \mathrm{U},{ }^{238} \mathrm{U}\right.$ or $\left.{ }^{239} \mathrm{Pu}\right)$ [7]. Some of these radioactive isotopes have already demonstrated its usefulness in different medical applications as will be discussed in chapter 2.1.

Due to its potential application in a diversity of research areas strontium has been the focus of interest in several studies as can be seen in recent reviews. Review papers have been published in 2004 on the biological role of strontium [1] and in 2006 on the biogeochemistry of strontium [6]. From to 2005 to present several reviews including, but not exclusively, strontium isotopic analysis can be found in the literature, focused on different subjects, such as geochemistry, ecogeochemistry and archaeology [25-27] as well as analytical methodologies [28-30]. Food authentication presents itself as the application with most review papers on the subject of stable isotopic techniques. This is most likely due to consumer's growing interest and concern towards food safety as well as current legislation. Although strontium is mentioned in some of such papers [31-36] the remaining are restricted to Isotope Ratio Mass Spectrometry (isotopic composition of light elements) leaving out the non-traditional heavier isotopes [37-40]. Most reviews published have mainly aimed at the different applications of stable isotope analysis. 
107 Based on these we will in addition compile a database of values for different biological

108 matrices providing a valuable tool for future works.

109 The isotope ratio analysis data treatment is just as important as the analytical

110 techniques employed in the isotope ratio measurements. Several statistical tools have

111 already been described in previous papers [31,32,41] and therefore the topic is out of

112 the scope of the current work.

113 Over the last 4 years an increasing number of studies have been published covering

114 the use of strontium isotopes in distinct areas of biological research. This review

115 focuses on strontium and its relationships with biological structures as well as the

116 potential applications of its isotopes in biological sciences and correlated scientific

117 fields. We will consider the application of strontium isotope ratios in health and

118 medicine as well as in fields associated with biological samples, namely food

119 authentication, archaeology and forensic sciences, reviewing scientific papers

120 published in the last five years (2012 to 2016), and therefore covering a time period for

121 which the topic has not yet been discussed. This wide approach based on strontium

122 can be helpful as support of a more integrated research in biological related sciences.

1.2 Biological role and toxicity

125

126 Strontium is considered a non-essential trace element for humans [42]. In fact, there are only two organisms for which its biological role has been identified, both are biominerals. The Acantharea, is a small group of protists that precipitate a strontium salt as a major exoskeleton constituent, celestite (strontium sulfate, $\mathrm{SrSO}_{4}$ ) [43]. The second is a cyanobacterium that forms intracellular amorphous carbonate inclusions containing strontium together with calcium, magnesium and barium, as benstonite [44]. These biominerals can provide information on the metabolism of those organisms, as well as on the biogeochemical cycles of strontium or past environmental conditions. For instances, the $\mathrm{Sr} / \mathrm{Ca}$ value of corals is related to the temperature of water during growth [45].

Strontium and calcium have similar atomic radius, 215 and $197 \mathrm{pm}$, respectively, and close chemical properties [46]. Also strontium is the sixteenth most abundant element in the Earth's crust [47] and the ninth in seawater [48], with average values of 200-300 $\mathrm{mg} / \mathrm{kg}$ and $8 \mathrm{mg} / \mathrm{kg}$, respectively [6,24]. For such reasons, although apparently without a biological role, strontium is found in several biological matrices. In table 1 , values of strontium in some biological samples are presented. 
Table 1 Strontium values in biological samples $(\mathrm{mg} / \mathrm{kg})$.

\begin{tabular}{|c|c|c|}
\hline Matrix & $\begin{array}{c}\text { Values } \\
(\mathrm{mg} / \mathrm{kg})\end{array}$ & \multirow{2}{*}{ Reference } \\
\hline Bread & $0.607-1.53$ & \multirow{2}{*}{} \\
\hline Vegetables & $0.007-12.6$ & \multirow{2}{*}[7]{} \\
\hline Cereals & $0.376-1.3$ & \multirow{2}{*}{ [49] } \\
\hline Fish & 3.5 & \\
\hline Milk & $0.128-0.432$ & {$[50]$} \\
\hline Eggs & $0.345-0.792$ & {$[51,52]$} \\
\hline Olive oil & $0.002-0.014$ & {$[53]$} \\
\hline Human hair & $0.7-45.1$ & \\
\hline Tooth enamel & $332-826$ & \\
\hline
\end{tabular}

145 The main pathway of exposure to strontium for humans is through the inhalation of aerosols and the ingestion of water and food [24], in particular of cereals and vegetables. It is estimated that a typical diet provides 2 to $4 \mathrm{mg}$ of strontium per day [1]. Strontium and calcium present similar distributions in the human body, with approximately $99 \%$ of the total body burden in the skeleton [24]. However, the total amount of strontium in human bodies is much smaller than calcium. While calcium represents $1.4 \%$ of total body mass, strontium accounts for only $0.00044 \%$ [1]. Several factors contribute to this, namely that calcium is more easily absorbed than strontium, the renal excretion of the last is three times higher than the former [1], and a much higher daily intake of calcium over strontium [7,54]. In addition, strontium suffers biopurification, i.e. for the next highest trophic level the $\mathrm{Sr} / \mathrm{Ca}$ is reduced by a factor of five [6] and humans do not belong to the lowest trophic levels.

157 Although some strontium salts have been identified as genotoxic human carcinogens, 158 namely strontium chromate $\left(\mathrm{CrO}_{4} \mathrm{Sr}\right)$ used in the production of pigments, the toxicity is not caused by strontium but from hexavalent chromium [24]. According to the Agency for Toxic Substances and Disease Registry (ATSDR) there is no direct evidence of toxicity by stable strontium isotopes in humans under normal environmental exposures. A minimal risk level for intermediate duration oral exposure to stable strontium is 2 $\mathrm{mg} /(\mathrm{kg} . \mathrm{bw} . \mathrm{day})$ [24]. 
164 On the other hand, exposure to radioactive strontium isotopes can lead to pathogenic

165 states, such as necrotic lesions and bone cancer, although the dose, the route of

166 exposure and the mass number of the isotope in question, affect the severity of the

167 disease. Within all of its radioactive isotopes ${ }^{90} \mathrm{Sr}$ is the most dangerous due to its

168 relatively long half-life (28.79 years [9]) and the bone-seeking properties of strontium

169 [24]. In fact, little interest on strontium in biology sciences occurred until the evidence

170 that ${ }^{90} \mathrm{Sr}$ could substitute calcium in bone [42].

171

172

1.3 Analytical methods

173

174

Isotope ratio mass spectrometry (IRMS) has been used for determination of lighter isotopic composition elements (e.g., $\mathrm{H}, \mathrm{C}, \mathrm{N}, \mathrm{O}$ and $\mathrm{S}$ ), while thermal ionization mass spectrometry (TIMS) was the unique technique that allowed the same purpose to the 'heavier' elements, with enough precision [55]. However, this technique is also characterized by some disadvantages, such as limited ionization efficiency for elements with ionization energy above $7.5 \mathrm{eV}$ [55], extensive sample preparation and long measurement time [56]. The development of the multicollector inductively coupled plasma mass spectrometry (MC ICP-MS) brought several advantages over TIMS, namely high ionization yield and versatile sample introduction systems well established in ICP spectrometry and also, since isotopic determinations occur simultaneously, no time-dependent mass fractionation as observed in TIMS analysis [57]. On the other hand TIMS is less prone to matrix effects and presents lower instrumental isotopic fractionation [27]. The radioactive decay of isotopes leads to extremely low differences in isotopic compositions, thus few techniques are capable of correctly performing isotope ratio measurements. Developments in the analytical instruments have provided high precision and accuracy measurement which are a fundamental requirement for the widespread use of isotope ratios in different applications [41]. Both TIMS and MC ICP-MS have become advanced techniques for the measurement of non-traditional isotopic compositions at high precision, namely strontium.

193 Recently the hypothesis of measuring the ${ }^{87} \mathrm{Sr} /{ }^{86} \mathrm{Sr}$ as part of proton activation analyses method by using a proton accelerator was tested [58]. Results were compared with TIMS and, although still at an early stage, were similar for SRM 987. Another new technique proposed for isotope measurements of several elements in cosmochemistry is the Chicago Instrument for Laser Ionization (CHILI). Still under optimization CHILI, which has been designed for the analysis of small samples, has produced its first meaningful scientific results for presolar grains [59]. 
200 Since our main goal is to present applications of strontium and its isotopes in biological

201 related fields as well as the analytical results obtained in such studies, a deeper

202 analysis of the analytical challenges associated with either technique is beyond the

203 scope of this review. The subject has already been approached by different authors

204 and thus will not be further developed here [11,27,36,60,61].

205 Strontium isotope ratios have been used over the past 40 years for geochronology or

206 as a geochemical tracers for past geological processes, such as the chemical

207 differentiation of the Earth, with special emphasis to ${ }^{87} \mathrm{Sr} /{ }^{86} \mathrm{Sr}$ ratio $[62,63]$. Nowadays

208 radiogenic isotopes are at the basis of various biological applications as presented in

209 the following chapters.

210

211 2. Applications in biological related fields

212

$213 \quad 2.1$ Medical applications

214

215 Even though strontium is considered without any biological role in humans some of its

216 radioactive isotopes have proven to be useful in medical applications. The mode of

217 decay as well as their half-life determines both toxicity and potential for medical

218 applications.

219 Since strontium and calcium have a similar behaviour in the human body, including the 220 same mechanisms for absorption from the gastrointestinal tract, bone accumulation, 221 blood clotting, muscular contraction, as shown in many pharmacological investigations 222 with isolated cells or organs, the response to stimulation tends to be weaker because 223 strontium is not so well adapted to these purposes [1,42] Both strontium and calcium 224 are excreted primarily in the urine. Strontium radioactive isotopes are used in several 225 medical applications. ${ }^{82} \mathrm{Sr}$ is used in nuclear medicine for the preparation of ${ }^{82} \mathrm{Rb}$ to 226 positron-emission tomography (PET) in cardiovascular diseases diagnosis [64], ${ }^{85} \mathrm{Sr}$ in 227 radiological imaging of bones [24] and metabolic studies [1], ${ }^{89} \mathrm{Sr}$, that emits $\beta$ -

228 radiation, relieves pain, improving the quality of life of patients with bone metastases

229 [65] and low levels of ${ }^{90} \mathrm{Sr}$ irradiation prevents the reappearance of primary pterygia 230 [66].

231 In addition to its radioactive isotopes also stable strontium has been used in the

232 treatment of illness. The drug strontium ranelate (Protelos/Osseor), which is composed

233 by an organic moiety (ranelic acid) and two atoms of stable nonradioactive strontium

234 [67], has shown promising results in the treatment of post-menopausal osteoporosis, 
by decreasing the risk of fractures. However, due to side effects, the European Medicines Agency has recommended its use only in severe osteoporosis, for postmenopausal women and adult men at high risk of fracture, and without a previous history of heart or circulatory problems [68]. In addition, its administration to patients with knee osteoarthritis has been described as a milestone, since it has produced clinically meaningful effects on pain relief and physical functions [69].

\subsection{Food provenance}

244

Radioactive decay in rocks of different ages leads to different isotope ratios in the labile fraction of the soils; the absorption of the isotopes by plants is reflected in the final food products, giving a unique isotopic signature associated with a geographical provenance, known as isotopic fingerprinting.

249 In recent years, it has been noticeable a growing interest by consumers on the 250 geographical origin of the food that they eat, to avoid serious foodborne diseases [13].

251 Furthermore, the European legislation on regional food protection considers the 252 authenticity as a quality criteria for the food, and its ingredients, subject that has gained 253 increasing importance over the past years [31].

254 Plants absorb elements mainly through their roots; therefore isotope ratios of plants depend both on the soil and climate conditions in which they grow. These reflect themselves on the isotopic composition of the final products, providing a unique fingerprint for studying traceability of numerous foodstuffs. The development of new advanced analytical techniques, over the last ten years, made possible the determination of the geographic origin of most foodstuffs through the analysis of its elemental and isotopic compositions [13]. Multi-isotope or single-isotope ratios can provide unique and representative fingerprints, thus making it possible to discriminate the origin of food samples.

263 Strontium is usually easily absorbed from soil by plants, even though there is no direct evidence of any metabolic function of the element in plants [7]. Hence, the strontium isotopic signature is a fingerprint representative of the soil on which plants were cultivated. It has been shown for rice and hot peppers, that the contribution of bedrock and the silicate fraction of the soil to bioavailable strontium is insignificant, while the composition of the water and the exchangeable fraction of the soil have the higher influence on bioavailable strontium [70]. On the other hand, Song et al. showed, 
271 fractions, strontium isotope ratios of bedrocks were maintained in soils and plants [71],

272 Once absorbed, strontium is distributed to other parts of the plant such as leaves or

273 fruits, turning it into a constituent of foodstuffs and thus entering the food web [7].

274 Subsequently, examples of foodstuffs will be presented.

275 Wine is most likely the food commodity with the highest number of studies in the area

276 of geographical origin authentication. This is probably due to the strict regulation of this

277 market and to the high commercial value achieved by wines from certain "Protected

278 Designation of Origin" (PDO). It has been recognized for a long time that the terroir

279 from which the wine is originated is essential to ensure its final quality. In 2013 a study

280 on Lambrusco wines, reported a good match between the strontium isotope ratios

281 monitored in the bio-available fraction of soils and their respective grape juices values

282 [72]. Even so a few years later, and although the strontium isotopic signature between

283 soils and Lambrusco wines were in agreement, it was not possible to distinguish

284 products from different years [73]. The same conclusions were obtained by Marchionni

285 et al. [74] since this study showed no influence of the production year or the

286 winemaking process in the ${ }^{87} \mathrm{Sr} /{ }^{86} \mathrm{Sr}$. Instead the authors concluded that the isotopic

287 fingerprint of wines is manly determined by the bioavailable fraction of the soils on

288 which the vineyards are settled.

289 Olive oils present sensory and nutritional properties often related to the geographical

290 origin and cultivar of the olive fruits employed [34]. It is a relevant constituent of the

291 Mediterranean diet and the European Union is responsible for $77 \%$ of olive oil world

292 production and $70 \%$ of consumption, mainly produced by Mediterranean countries [75].

293 However, only recently the first work using strontium isotopes to trace the origin of olive

294 oils was published [50]. Despite of a complex matrix with high fat and low strontium

295 content (caused by its ionic form), the authors developed and validated a method for

296 strontium extraction for seven samples.

297 Other matrices, such as cheese, exhibited a wide variation in the ${ }^{87} \mathrm{Sr} /{ }^{86} \mathrm{Sr}$ which

298 allowed to distinguish products from different farmers [76] while in Italian tomatoes and

299 tomato derived products strontium isotope ratios where sufficiently discriminative to

300 successfully distinguish these from similar Chinese products [77].

301 In 2014 the origin of peanuts from different countries was compared exhibiting ratios of

$302{ }^{87} \mathrm{Sr} /{ }^{86} \mathrm{Sr}$ below 0.702 [78], as can be seen in the table 2. However, such value has

303 been considered the lowest ${ }^{87} \mathrm{Sr} /{ }^{86} \mathrm{Sr}$ value currently found in Earth [6,12] and in

304 accordance with the results found in our review, some relevant questions may be

305 raised regarding the methodology applied. First and most importantly, is the analytical

306 method used, inductively coupled plasma - quadrupole mass spectrometry (ICP-Q- 
307 MS). It is generally accepted that, unlike MC ICP-MS or TIMS (precision better than

$308 \quad 0.005 \%$ ), ICP-Q-MS does not provide a high enough precision for the determination of 309 isotope ratios since at best it is $0.05 \%$ [79]. Moreover, the extraction of strontium from

310 matrix was carried out using $\mathrm{HNO}_{3}$ combined with sonication, while the most common

311 procedure is solid phase extraction following digestion, which could have led to some

312 bias in sample preparation.

313 Table 2 summarizes recent results of the strontium isotopic signature from food

314 sources.

$315{ }^{87} \mathrm{Sr} /{ }^{86} \mathrm{Sr}$ has also been employed in identifying the origin of fish as well as to

316 distinguish between farmed and wild fishes [16,80-82]. On the basis of such

317 application is the fact that, similarly to what happens in human skeletal tissues (see 2.3

318 Bioarchaeology), strontium replaces for calcium in otoliths and scales. Comparing the

319 strontium isotope ratios in such calcified tissues to the values of surrounding waters

320 provides a tool to identify the origin and migration habits of such animals. While otoliths

321 are the most common structure for determining the origin of fish [81-88] scales and fin

322 rays have been suggested as a promising nonlethal alternative for provenance studies $323[16,80,89]$. 
Table 2 Application of strontium isotope ratios in food authentication.

\begin{tabular}{|c|c|c|c|c|c|c|}
\hline \multirow{2}{*}{ Matrices } & \multirow{2}{*}{ Number of samples } & \multicolumn{3}{|c|}{ Range of ${ }^{87} \mathrm{Sr} /{ }^{86} \mathrm{Sr}$} & \multirow{2}{*}{$\begin{array}{l}\text { Analytical } \\
\text { technique }\end{array}$} & \multirow{2}{*}{ Reference } \\
\hline & & Minimum & Maximum & Mean & & \\
\hline \multirow{2}{*}{$\begin{array}{l}\text { Chinese } \\
\text { cabbage }\end{array}$} & 160 (cabbage) & 0.70814 & 0.72018 & 0.71139 & \multirow{2}{*}{ TIMS } & \multirow{2}{*}[90]{} \\
\hline & 36 (soil) & 0.70639 & 0.72836 & 0.71649 & & \\
\hline \multirow{2}{*}{ Kimchi } & 29 (unwashed Kimchi) & n.a. & n.a. & 0.71060 & \multirow{2}{*}{ MC ICP-MS } & \multirow{2}{*}{ [91] } \\
\hline & 29 (washed Kimchi) & n.a. & n.a. & 0.71099 & & \\
\hline \multirow{3}{*}{$\begin{array}{l}\text { Hot pepper } \\
\text { and rice }\end{array}$} & 77 (plants) & 0.70803 & 0.73909 & 0.71712 & \multirow{3}{*}{ MC ICP-MS } & \multirow{3}{*}{ [70] } \\
\hline & 137 (soils) & 0.70646 & 0.76470 & 0.71891 & & \\
\hline & 191 (water) & 0.70698 & 0.75753 & 0.71881 & & \\
\hline Rice & 350 (rice) & 0.704 & 0.721 & 0.709 & HR-ICP-MS & [92] \\
\hline Beef & 206 (beef) & 0.70628 & 0.71649 & 0.70978 & TIMS & [93] \\
\hline Onions & 139 (onions) & 0.70395 & 0.72099 & 0.70987 & MC ICP-MS & [94] \\
\hline Olive oil & 7 & 0.70727 & 0.70914 & 0.70836 & TIMS & {$[50]$} \\
\hline Tomato & $\begin{array}{l}118 \text { (tomato and tomato } \\
\text { derived products) }\end{array}$ & 0.70793 & 0.71051 & 0.70899 & TIMS & [77] \\
\hline \multirow{2}{*}{ Wine } & 10 (soils) & 0.70772 & 0.71115 & 0.71097 & \multirow{2}{*}{ TIMS } & \multirow{2}{*}{ [95] } \\
\hline & 30 (must) & 0.70706 & 0.71266 & 0.70987 & & \\
\hline
\end{tabular}




\begin{tabular}{|c|c|c|c|c|c|c|}
\hline & 186 & 0.70839 & 0.70964 & n.a. & MC ICP-MS & [73] \\
\hline & 22 & 0.7130 & 0.7175 & n.a. & ICP-Q-MS & [96] \\
\hline & 45 & 0.70679 & 0.71131 & 0.70865 & TIMS & [74] \\
\hline & 16 (wine) & 0.70886 & 0.71040 & n.a. & \multirow{3}{*}{ MC ICP-MS } & \multirow{3}{*}{ [72] } \\
\hline & 16 (branch) & ca. 0.7086 & ca. 0.7108 & n.a. & & \\
\hline & 80 (soils) & 0.70867 & 0.71300 & n.a. & & \\
\hline & 4 (grape) & 0.70894 & 0.71062 & 0.70984 & \multirow{5}{*}{ TIMS } & \multirow{5}{*}{ [97] } \\
\hline & 15 (must) & 0.70899 & 0.71038 & 0.70953 & & \\
\hline & 23 (wine) & 0.70898 & 0.71057 & 0.7096 & & \\
\hline & 6 (soil) & 0.70995 & 0.71142 & 0.71058 & & \\
\hline & 12 (rocks) & 0.70885 & 0.71796 & 0.71147 & & \\
\hline & 6 (wine) & 0.708 & 0.715 & 0.710 & ICP-Q-MS & [98] \\
\hline & 16 (grapes) & 0.70945 & 0.71522 & 0.71163 & \multirow{4}{*}{ TIMS } & \multirow{4}{*}{ [99] } \\
\hline & 17 (wines) & 0.70988 & 0.71546 & 0.71206 & & \\
\hline & 13 (bulk soil) & 0.71203 & 0.73418 & 0.72002 & & \\
\hline & 9 (labile soil) & 0.70979 & 0.71546 & 0.71182 & & \\
\hline \multirow{3}{*}{ Cheese } & 14 (cheese) & 0.70950 & 0.71360 & 0.71156 & \multirow{3}{*}{ TIMS } & \multirow{3}{*}{ [76] } \\
\hline & 12 (milk) & 0.70961 & 0.71425 & 0.71192 & & \\
\hline & 6 (soil) & 0.70955 & 0.71453 & 0.71185 & & \\
\hline Coffee beans & 13 & 0.70472 & 0.72027 & 0.70781 & MC ICP-MS & {$[100]$} \\
\hline
\end{tabular}




\begin{tabular}{ccccccc}
\hline Tea leaves & 14 & 0.70482 & 0.71462 & 0.70965 & MC ICP-MS & [101] \\
\hline Peanut & 200 & $c a .0 .695$ & $c a .0 .726$ & $c a .0 .712$ & ICP-Q-MS & {$[78]$} \\
\hline Poultry & 105 & 0.70706 & 0.71114 & 0.70865 & TIMS & {$[102]$} \\
\hline Beer & 80 & $c a .0 .707$ & $c a .0 .718$ & n.a. & MC ICP-MS & {$[103]$} \\
\hline Wheat & 80 & $0.7073^{\mathrm{a}}$ & $0.7089^{\mathrm{a}}$ & $0.7081^{\mathrm{a}}$ & TIMS & {$[104]$} \\
\cline { 2 - 6 } & 54 & 0.711 & 0.712 & 0.71154 & TIMS & {$[105]$} \\
\hline Honey & 79 & $0.7125^{\mathrm{a}}$ & $0.7172^{\mathrm{a}}$ & $0.7152^{\mathrm{a}}$ & TIMS & {$[106]$} \\
\hline
\end{tabular}

326

a mean value of a region. Original values are not supplied

327

328 


\subsection{Bioarchaeology}

330

331

332

333

334

335

336

337

338

339

340

341

342

343

344

345

346

347

348

349

350

351

352

353

354

355

356

357

358

359

360

361

362

363

364

In animals the strontium isotopic composition is a function of the surrounding environment and dietary habits [5]. ${ }^{87} \mathrm{Sr} /{ }^{86} \mathrm{Sr}$ values of skeletal tissues have been used to study dietary (in conjunction with other isotopic systems) and migration patterns of ancient human and animal populations. In fact the employment of stable isotopes ratios in archaeological studies has increased to such an extent in the latest years that they are now regarded as routine analysis in researches dealing with such topics [107].

The similarities between calcium and strontium's chemical characteristics explains the accumulation of strontium in bones and teeth [both materials in mammals are mainly hydroxyapatite, $\mathrm{Ca}_{5}\left(\mathrm{PO}_{4}\right)_{3}(\mathrm{OH})$ ]. However, unlike bone, that rebuilds itself through life, as part of the body's maintenance, dental enamel formed during infancy remains unaltered throughout the years, therefore its isotopic signature is representative of the diet during the formation period of the teeth [108-110]. Post mortem diagenesis of bones and teeth is defined as alteration occurring in the physical, chemical or microstructural composition of such tissues following its deposition in the environment [111]. Enamel is known to be less prone to post mortem diagenesis than dentine and bone, which have been considered unreliable markers of strontium since they mainly reflect the bioavailable strontium of burial place $[112,113]$. Since not all teeth are formed at the same time, isotope ratios of the enamel from different teeth will represent different periods of a person's life [114]. It is for this reason that the analysis of strontium isotopes in tooth enamel from burial populations, has been widely applied in the last years to study human migrations, hunting, trading areas and diet habits amongst ancient populations [109,115-117]. It is not expectable to find an absolute value for a certain geographical region. Instead a range of bioavailable ${ }^{87} \mathrm{Sr} /{ }^{86} \mathrm{Sr}$ in the area of interest should be considered as baseline [110]. Two possible approaches have been suggested either by resorting to local fauna remains or the average of human dental enamel [118].In Germany the comparison between water, soils, floral and faunal samples, both modern and archaeological, showed that contemporary water and vegetation samples, particularly tree leaves, served as the best proxy for local bioavailable strontium [119]. More recently it has been proposed the application of Bayesian mixing analysis as a statistical technique to understand results from isotope ratio analysis, which according to the authors allows interpreting strontium values within a context [120].

A compilation of scientific results using strontium isotopes in diet and migratory patterns of ancient human populations is presented in table 3. 
365 Table 3 Application of strontium isotope ratios in migration and diet studies.

\begin{tabular}{|c|c|c|c|c|c|c|c|}
\hline \multirow{2}{*}{ Matrices } & \multirow{2}{*}{ Number of samples } & \multicolumn{3}{|c|}{ Range of ${ }^{87} \mathrm{Sr} /{ }^{86} \mathrm{Sr}$} & \multirow{2}{*}{ Analytical technique } & \multirow{2}{*}{ Geographical area } & \multirow{2}{*}{ Reference } \\
\hline & & Minimum & Maximum & Mean & & & \\
\hline \multirow{17}{*}{ Tooth enamel } & 1 (human) $^{\mathrm{a}}$ & 0.7202 & 0.7211 & 0.7207 & LA- MC ICP-MS & South Africa & [121] \\
\hline & 6 (human) & 0.70941 & 0.71057 & 0.70980 & \multirow{2}{*}{ MC ICP-MS } & \multirow{2}{*}{ Mongolia } & \multirow{2}{*}[122]{} \\
\hline & 20 (faunal) & 0.70817 & 0.70831 & 0.70824 & & & \\
\hline & 86 (human) & 0.70766 & 0.70850 & 0.70812 & TIMS & Guatemala & [123] \\
\hline & 19 (human) & 0.7090 & 0.7109 & 0.7099 & \multirow{2}{*}{ TIMS } & \multirow{2}{*}{ Isle of Man } & \multirow{2}{*}{ [124] } \\
\hline & 3 (plants) & 0.7091 & 0.7113 & 0.7103 & & & \\
\hline & 29 (human) & 0.70809 & 0.71167 & 0.70920 & TIMS & England & [125] \\
\hline & 33 (human) & 0.7092 & 0.7134 & 0.7104 & TIMS & Wales & [126] \\
\hline & 133 (human) & 0.70837 & 0.71085 & 0.70944 & \multirow{2}{*}{ MC ICP-MS } & United States of & \multirow{2}{*}{ [108] } \\
\hline & 20 (faunal) & 0.70888 & 0.70968 & 0.70933 & & America & \\
\hline & 15 (human) & 0.7076 & 0.7088 & 0.7084 & MC ICP-MS & Vanuatu & [127] \\
\hline & 55 (human) & 0.70670 & 0.72073 & 0.71012 & \multirow{2}{*}{ MC ICP-MS } & \multirow{2}{*}{ Portugal } & \multirow{2}{*}{ [109] } \\
\hline & 22 (faunal) & 0.70550 & 0.71328 & 0.71031 & & & \\
\hline & 100 (human) & 0.70818 & 0.71066 & 0.70886 & \multirow{2}{*}{ TIMS } & \multirow{2}{*}{ South eastern Arabia } & \multirow{2}{*}{ [115] } \\
\hline & 104 (faunal) & 0.70808 & 0.71042 & 0.70860 & & & \\
\hline & 53 (human) & 0.7086 & 0.7169 & 0.7101 & TIMS & Germany & [128] \\
\hline & 32 (human) & 0.7046 & 0.7117 & n.a. & TIMS & Honduras & [110] \\
\hline
\end{tabular}




\begin{tabular}{|c|c|c|c|c|c|c|c|}
\hline & 77 (human) & 0.7113 & 0.72802 & 0.71618 & \multirow{3}{*}{ TIMS } & \multirow{3}{*}{$\begin{array}{l}\text { Pakistan } \\
\text { India }\end{array}$} & \multirow{3}{*}[53]{} \\
\hline & 21 (faunal) & 0.71471 & 0.72112 & 0.71771 & & & \\
\hline & 3 (sediments) & 0.71553 & 0.71594 & 0.71565 & & & \\
\hline & 15 (human) & 0.70607 & 0.70863 & 0.70781 & TIMS & Belize & [129] \\
\hline & 109 (human) & 0.7077 & 0.7391 & 0.7157 & \multirow{2}{*}{ TIMS } & Island of Oland & \multirow{2}{*}{ [120] } \\
\hline & 21 (faunal) & 0.7098 & 0.7215 & 0.7155 & & (Baltic) & \\
\hline & 127 (domestic animals) & 0.7080 & 0.7101 & 0.7093 & MC ICP-MS & India & [130] \\
\hline & 20 (human) & 0.70828 & 0.71236 & 0.7096 & TIMS & $\begin{array}{l}\text { Londinium (London, } \\
\text { England in the } \\
\text { Roman period) }\end{array}$ & [131] \\
\hline & 117 (human) & 0.70822 & 0.71621 & 0.71093 & MC ICP-MS & Germany & [132] \\
\hline & 105 (human) & 0.70100 & 0.71398 & 0.70911 & \multirow{2}{*}{ TIMS } & \multirow{2}{*}{ Italy } & \multirow{2}{*}{ [133] } \\
\hline & 2 (faunal) & 0.70933 & 0.71031 & $0-70982$ & & & \\
\hline & 14 (human) & 0.71027 & 0.71359 & 0.71168 & TIMS & Portugal & [134] \\
\hline & 34 (human) & 0.70573 & 0.71069 & 0.70764 & MC ICP-MS & Peru & [135] \\
\hline & 41 (human) & 0.70589 & 0.74985 & 0.71984 & MC ICP-MS & Brazil & [136] \\
\hline & 35 (human) & 0.70794 & 0.71262 & 0.70905 & \multirow{2}{*}{ TIMS } & \multirow{2}{*}{ England } & \multirow{2}{*}{ [137] } \\
\hline & 3 (faunal) & 0.70774 & 0.71059 & 0.70885 & & & \\
\hline & 8 (human) & 0.71100 & 0.71956 & 0.71449 & \multirow{2}{*}{ TIMS } & \multirow{2}{*}{ Estonia } & \multirow{2}{*}{ [138] } \\
\hline & 7 (faunal) & 0.71056 & 0.71594 & 0.71217 & & & \\
\hline & 2 (human) & 0.71100 & 0.71106 & 0.71103 & TIMS & England & [139] \\
\hline Tooth enamel and & 12 (human enamel) & 0.70820 & 0.71314 & 0.71060 & MC ICP-MS & France & [140] \\
\hline
\end{tabular}




\begin{tabular}{|c|c|c|c|c|c|c|c|}
\hline bone & 13 (human bones) & 0.70976 & 0.71226 & 0.71064 & & & \\
\hline & 60 (human enamel) & 0.70849 & 0.70925 & 0.70890 & \multirow{3}{*}{ TIMS } & \multirow{3}{*}{ Japan } & \multirow{3}{*}[141]{} \\
\hline & 12 (human bones) & 0.70871 & 0.70897 & 0.70883 & & & \\
\hline & 42 (plants) & 0,71071 & 0.70909 & 0.70696 & & & \\
\hline & 12 (human enamel) & 0.70824 & 0.71099 & 0.71017 & \multirow{4}{*}{ MC ICP-MS } & \multirow{4}{*}{ Ireland } & \multirow{4}{*}[142]{} \\
\hline & 10 (human bones) & 0.70906 & 0.70974 & 0.70928 & & & \\
\hline & 2 (faunal enamel) & 0.70909 & 0.70914 & 0.70912 & & & \\
\hline & 9 (faunal bones) & 0.70925 & 0.70995 & 0.70950 & & & \\
\hline & 19 (human enamel) & 0.70561 & 0.70747 & 0.70641 & \multirow{2}{*}{ MC ICP-MS } & \multirow{2}{*}{ Peru } & \multirow{2}{*}{ [143] } \\
\hline & 12 (human bones) & 0.70617 & 0.70690 & 0.70661 & & & \\
\hline & 1 (human enamel) & 0.70883 & 0.70883 & 0.70883 & \multirow{3}{*}{ MC ICP-MS } & \multirow{3}{*}{ Chile } & \multirow{3}{*}[144]{} \\
\hline & 2 (human bones) & 0.70745 & 0.70806 & 0.70776 & & & \\
\hline & 2 (plants) & 0.70700 & 0.71031 & 0.70866 & & & \\
\hline & 115 (human enamel) & 0.70911 & 0.71067 & 0.70987 & \multirow{2}{*}{ MC ICP-MS } & \multirow{2}{*}{ Hungary } & \multirow{2}{*}{ [145] } \\
\hline & 45 (human bones) & 0.70919 & 0.71057 & 0.70997 & & & \\
\hline & 45 (human enamel) & 0.70758 & 0.71252 & 0.70829 & TIMS and & \multirow{2}{*}{ Chile } & \multirow{2}{*}{ [146] } \\
\hline & 12 (human bones) & 0.70776 & 0.70886 & 0.70817 & MC ICP-MS & & \\
\hline & 22 (gazelle enamel) & 0.70761 & 0.70842 & 0.70813 & \multirow{3}{*}{ TIMS } & \multirow{3}{*}{ Anatolia (Turkey) } & \multirow{3}{*}[147]{} \\
\hline & 22 (gazelle bone) & 0.70793 & 0.70825 & 0.70813 & & & \\
\hline & 6 (faunal bone) & 0.70810 & 0.70827 & 0.70817 & & & \\
\hline & 14 (human enamel) & 0.70668 & 0.71006 & 0.70935 & \multirow{2}{*}{ TIMS } & \multirow{2}{*}{ China } & \multirow{2}{*}[148]{} \\
\hline & 11 (human bone) & 0.70887 & 0.70992 & 0,70926 & & & \\
\hline
\end{tabular}




\begin{tabular}{|c|c|c|c|c|c|c|}
\hline 66 (human enamel) & 0.70829 & 0.71431 & 0.70970 & \multirow{3}{*}{ TIMS } & \multirow{3}{*}{ Hungary } & \multirow{3}{*}[149]{} \\
\hline 2 (human bone) & 0.70957 & 0.70995 & 0.70976 & & & \\
\hline 22 (plants) & 0.70688 & 0.71255 & 0.70938 & & & \\
\hline 6 (human enamel) & 0.70669 & 0.70785 & 0.70693 & \multirow{3}{*}{ MC ICP-MS } & \multirow{3}{*}{$\begin{array}{c}\text { United States of } \\
\text { America }\end{array}$} & \multirow{3}{*}[150]{} \\
\hline 3 (human bones) & 0.70630 & 0.70642 & 0,70635 & & & \\
\hline 7 (faunal bones) & 0.70663 & 0.70691 & 0.70675 & & & \\
\hline 38 (human enamel) & 0.710 & 0.733 & 0.717 & \multirow{3}{*}{ TIMS } & \multirow{3}{*}{ Sweden } & \multirow{3}{*}[151]{} \\
\hline 3 (faunal enamel) & 0.722 & 0.739 & 0.728 & & & \\
\hline 3 (faunal bones) & 0.728 & 0.737 & 0.734 & & & \\
\hline 36 (human enamel) & 0.70891 & 0.70957 & 0.70920 & \multirow{3}{*}{ MC ICP-MS } & \multirow{3}{*}{ Spain } & \multirow{3}{*}{ [152] } \\
\hline 3 (human bones) & 0.70933 & 0.70946 & 0.70938 & & & \\
\hline 2 (faunal enamel) & 0.70921 & 0.70924 & 0.70923 & & & \\
\hline 3 (human enamel) & 0.70846 & 0.70896 & 0.70868 & \multirow[b]{2}{*}{ MC ICP-MS } & \multirow[b]{2}{*}{ Ireland } & \multirow[b]{2}{*}{ [153] } \\
\hline $\begin{array}{c}17 \text { (human calcined } \\
\text { bones) }\end{array}$ & 0.70660 & 0.71362 & 0.70896 & & & \\
\hline 32 (human enamel) & 0.70827 & 0.70880 & 0.70860 & \multirow{4}{*}{ TIMS } & \multirow{4}{*}{ Italy } & \multirow{4}{*}[154]{} \\
\hline 33 (human bone) & 0.70820 & 0.70871 & 0.70855 & & & \\
\hline 4 (faunal enamel) & 0.70858 & 0.70863 & 0.70860 & & & \\
\hline 7 (faunal bone) & 0.70817 & 0.70856 & 0.70838 & & & \\
\hline 13 (human enamel) & 0.70868 & 0.71234 & 0.70959 & \multirow{4}{*}{ MC ICP-MS } & \multirow{4}{*}{ Spain } & \multirow{4}{*}[155]{} \\
\hline 29 (human bone) & 0.70864 & 0.70946 & 0.70896 & & & \\
\hline 1 (faunal enamel) & 0.70890 & 0.70890 & 0.70890 & & & \\
\hline 6 (faunal bone) & 0.70861 & 0.70901 & 0.70884 & & & \\
\hline
\end{tabular}




\begin{tabular}{|c|c|c|c|c|c|c|c|}
\hline & 34 (human enamel) & 0.70997 & 0.71103 & 0.71043 & \multirow{2}{*}{ TIMS } & Central Asia & \multirow{2}{*}{ [156] } \\
\hline & 12 (faunal bones) & 0.71030 & 0.71053 & 0.71043 & & (Pamir Plateau) & \\
\hline & 36 (human enamel) & 0.70763 & 0.71384 & 0.71127 & \multirow{3}{*}{ MC ICP-MS } & \multirow{3}{*}{ Germany } & \multirow{3}{*}{ [157] } \\
\hline & 8 (human bones) & 0.70941 & 0.71008 & 0.70974 & & & \\
\hline & 3 (faunal bones) & 0.70900 & 0.70922 & 0.70914 & & & \\
\hline \multirow{18}{*}{$\begin{array}{c}\text { Tooth enamel and } \\
\text { dentine }\end{array}$} & 76 (human enamel) & 0.70725 & 0.71923 & 0.71296 & \multirow{2}{*}{ MC ICP-MS } & \multirow{2}{*}{ Germany } & \multirow{2}{*}{ [158] } \\
\hline & 5 (human dentine) & 0.70904 & 0.71416 & 0.71195 & & & \\
\hline & 7 (human enamel) & 0.70871 & 0.70956 & 0.70907 & \multirow{4}{*}{ TIMS } & \multirow{4}{*}{ England } & \multirow{8}{*}[159]{} \\
\hline & 4 (human dentine) & 0.70957 & 0.70981 & 0.70969 & & & \\
\hline & 7 (plants) & 0,70856 & 0.71041 & 0.70922 & & & \\
\hline & 4 (faunal) & 0.70804 & 0.70830 & 0.70821 & & & \\
\hline & 12 (human enamel) & 0.70816 & 0.71024 & 0.70941 & \multirow{2}{*}{ TIMS } & \multirow{2}{*}{ France } & \\
\hline & 4 (human dentine) & 0.70883 & 0.70993 & 0.70941 & & & \\
\hline & 5 (human enamel) & 0.70792 & 0.70999 & 0.70846 & \multirow{2}{*}{ TIMS } & \multirow{2}{*}{ Germany } & \\
\hline & 2 (human dentine) & 0.70789 & 0.70810 & 0.70710 & & & \\
\hline & 32 (human enamel) & 0.70798 & 0.71049 & 0.70962 & \multirow{2}{*}{ TIMS } & \multirow{2}{*}{ England } & \multirow{2}{*}{ [160] } \\
\hline & 8 (human dentine) & 0.70777 & 0.71002 & 0.70840 & & & \\
\hline & 28 (human enamel) & 0.70860 & 0.71200 & 0.70970 & \multirow{2}{*}{ TIMS } & \multirow{2}{*}{$\begin{array}{l}\text { United States of } \\
\qquad \text { America }\end{array}$} & \multirow{2}{*}{ [161] } \\
\hline & 28 (human dentine) & 0.70828 & 0.71080 & 0.70934 & & & \\
\hline & 23 (human enamel) & 0.70879 & 0.71645 & 0.71016 & \multirow{4}{*}{ TIMS } & \multirow{4}{*}{ Netherlands } & \multirow{4}{*}{ [117] } \\
\hline & 3 (human dentine) & 0.70902 & 0.71101 & 0.71021 & & & \\
\hline & 3 (faunal enamel) & 0.70901 & 0.70996 & 0.70936 & & & \\
\hline & 7 (plants) & 0.7113 & 0.7156 & 0.7134 & & & \\
\hline
\end{tabular}




\begin{tabular}{|c|c|c|c|c|c|c|c|}
\hline & 95 (cattle teeth) & 0.70786 & 0.71448 & 0.70965 & TIMS & England & [114] \\
\hline & 12 (human enamel) & 0.7076 & 0.7081 & 0.7079 & \multirow{2}{*}{ MC ICP-MS } & \multirow{2}{*}{ Jordan } & \multirow{2}{*}{ [162] } \\
\hline & 8 (human dentine) & 0.7077 & 0.7081 & 0.7079 & & & \\
\hline & 11 (human enamel) & 0.71061 & 0.71203 & 0.71134 & \multirow{4}{*}{ TIMS } & \multirow{4}{*}{ Libya } & \multirow{4}{*}{163} \\
\hline & 5 (faunal enamel) & 0.71085 & 0.71150 & 0.71161 & & & \\
\hline & 1 (faunal bone) & 0.71098 & 0.71098 & 0.71098 & & & \\
\hline & 3 (shells) & 0.71031 & 0.71129 & 0.71081 & & & \\
\hline & 19 (human enamel) & 0.70897 & 0.71329 & 0.71038 & \multirow{2}{*}{ TIMS } & \multirow{2}{*}{ Denmark } & \multirow{2}{*}{ [164] } \\
\hline & 6 (faunal) & 0.70927 & 0.71143 & 0.71039 & & & \\
\hline & 24 (human enamel) & 0.70740 & 0.70940 & 0.70838 & \multirow{4}{*}{ MC ICP-MS } & \multirow{4}{*}{ Germany } & \multirow{4}{*}[165]{} \\
\hline \multirow{10}{*}{ Various } & 8 (human dentine) & 0.70773 & 0.70868 & 0.70827 & & & \\
\hline & 7 (snail shell) & 0.70570 & 0.70947 & 0.70755 & & & \\
\hline & 11 (plants) & 0.70616 & 0.71087 & 0.70843 & & & \\
\hline & 21 (sheep enamel) & 0.70880 & 0.70961 & 0.70934 & \multirow{3}{*}{ TIMS } & \multirow{3}{*}{ Spain } & \multirow{3}{*}[166]{} \\
\hline & 3 (sheep dentine) & 0.70945 & 0.70955 & 0.70951 & & & \\
\hline & 2 (faunal) & 0.70951 & 0.70953 & 0.70952 & & & \\
\hline & 124 (faunal) & 0.70694 & 0.74614 & 0.71103 & \multirow{4}{*}{ MC ICP-MS } & \multirow{4}{*}{ Siberia } & \multirow{4}{*}{ [167] } \\
\hline & 179 (plants) & 0.70673 & 0.75225 & 0.71215 & & & \\
\hline & 60 (water) & 0.70711 & 0.77350 & 0.71386 & & & \\
\hline & 39 (human teeth) & 0.70801 & 0.72991 & 0.71496 & & & \\
\hline
\end{tabular}




\begin{tabular}{cccccc}
\hline 64 (human teeth) & 0.70888 & 0.71188 & 0.70950 & TIMS & Germany \\
8 (human bones) & 0.70901 & 0.70939 & 0.70919 & 0.70896 & Italy \\
5 (pig enamel) & 0.70888 & 0.70907 & 0 & \\
\hline
\end{tabular}

$366 \quad{ }^{a}$ the sample was analysed 14 times 
369 The joint analysis of oxygen and strontium isotopes evidenced the maritime trading

370 activities of populations from northern Netherlands during early medieval period,

371 revealed some possibilities about their diets [117]. In the same way the study of

372 strontium $\left({ }^{87} \mathrm{Sr} /{ }^{86} \mathrm{Sr}\right)$ and oxygen $\left(\delta^{18} \mathrm{O}\right)$, established that tooth pendants were

373 exchanged and/or transported over vast distances across the Caribbean [169]. On the

374 other hand, crossing strontium and lead (is not an essential metal, but as divalent ion

375 can replace calcium in some cases) isotopic data revealed dietary patterns of the Indus

376 Civilization (2600-1900 BC) which were unnoticeable by analysing each element

377 independently [53]. The same isotopic combination proved to be useful to distinguish

378 between natives and migrants of the ancient Roman London [131].The combination of

379 geochemical data from ${ }^{87} \mathrm{Sr} /{ }^{86} \mathrm{Sr}$ with ${ }^{14} \mathrm{C}, \delta^{13} \mathrm{C}$, and $\delta^{15} \mathrm{~N}$, evidenced the existence of

380 two different diets in a population from middle Holocene Early Bronze Age in Siberia,

381 corresponding to local and nonlocal individuals [170].

382 In marine products, unlike the Earth's crust, ${ }^{87} \mathrm{Sr} /{ }^{86} \mathrm{Sr}$ is constant throughout the oceans

383 at a given period of time. Isotope ratios of sea shells (composed by calcium salts,

384 mainly carbonates) reflect the seawater ratio at the time of their formation and are

385 useful tools in investigating the source of archaeological shell artefacts [6].

386 Strontium isotopes have proved to be a reliable biological marker in the study of past

387 human and animal migrations. Nonetheless the obtained results may be refined by

388 complementing with information from other isotopic system, either from light elements

$389[117,169]$, rare earth elements such as $\mathrm{Nd}[171,172]$ or even heavy elements like lead

$390 \quad([53])$

\section{$391 \quad 2.4$ Forensic science}

392

393 It is possible to determine the provenance of unidentified corpses by studying stable

394 isotopes in body tissue materials such as teeth, bones, hair and nails (table 4). These

395 provide information of different periods from childhood to death of a human [173].

396 However, in forensic sciences it is more common the use of light isotopes $(\mathrm{C}, \mathrm{H}, \mathrm{O}$ and

397 N) [174-176] rather than strontium isotopes most likely due to analytical challengesin

398 the preparation of samples associated with the metal.

399 In hair, the ${ }^{87} \mathrm{Sr} /{ }^{86} \mathrm{Sr}$ is not exclusively controlled by water or diet intake [52]. In fact,

400 both internal and external factors, diet and environment, respectively, have an

401 influence on the isotope ratio and must be taken into consideration when studying this

402 matrix. Hence, two potential methods have been suggested to separate endogenous

403 strontium from exogenous strontium [51]. However, there still isn't a consensual 
404 method for such purpose. Although Font et al. showed that the migrations across

405 geographical areas can stay recorded in scalp hair by the ${ }^{87} \mathrm{Sr} /{ }^{86} \mathrm{Sr}$ isotope depending

406 on a monthly timescale, however, further studies are recommended by the authors to

407 confirm this possibility [177].

408 For human skeletal remains, namely tooth enamel and bone, strontium isotopic

409 analyses allow to reduce potential areas of residence and even the total exclusion of

410 some regions, thus giving complementary information that may be clarified by other

411 techniques [118]. The use of multidisciplinary approaches is valuable in the

412 identification of human remains [178]. Tooth enamel is formed during childhood and,

413 unlike hair, is not influenced by exogenous sources of strontium, hence it is a good

414 marker for determining the place of birth or growing location during infancy. This was

415 verified in search of the provenance of two unidentified World War II casualties. The

416 combination of oxygen and strontium isotope ratios suggested the South Coast and/or

417 Central Eastern part of the United Kingdom as probable origin of these casualties

418 [179].

419 A more recent application of strontium in forensic sciences has been developed in the

420 last decade, as a tool to diagnose death by drowning [180-183] and even to be related

421 with different time-lapses of the agonal period [184]. Due to the high levels of this metal

422 in oceans, in seawater drowning the serum levels and mean concentration of strontium

423 in the right ventricle are significantly higher compared to the left ventricle. It has been

424 shown that serum levels of strontium are in fact a highly sensitive marker of water

425 aspiration, particularly in the case of seawater drowning, while in freshwater drowning

426 combining strontium with other trace elements increases the chances of a correct

427 diagnosis [183,185]. 
$430 \quad$ Table 4

431 Application of strontium isotope ratios in forensic sciences.

\begin{tabular}{|c|c|c|c|c|c|c|}
\hline \multirow{2}{*}{ Matrices } & \multirow{2}{*}{ Number of samples } & \multicolumn{3}{|c|}{ Range of ${ }^{87} \mathrm{Sr} /{ }^{86} \mathrm{Sr}$} & \multirow{2}{*}{$\begin{array}{l}\text { Analytical } \\
\text { technique }\end{array}$} & \multirow{2}{*}{ Reference } \\
\hline & & Minimum & Maximum & Mean & & \\
\hline & 11 (bulk hair) & 0.70909 & 0.71040 & 0.70968 & \multirow{3}{*}{ TIMS } & \multirow{3}{*}{ [52] } \\
\hline \multirow[t]{2}{*}{ Human hair } & 23 (hair strands) & 0.70990 & 0.71062 & 0.70965 & & \\
\hline & 8 (water) & 0.70801 & 0.71011 & 0.70922 & & \\
\hline Human hair & 22 & 0.70909 & 0.71469 & 0.712023 & MC ICP-MS & [51] \\
\hline Tooth enamel & 1 & 0.71103 & 0.71103 & 0.71103 & \multirow{2}{*}{ MC ICP-MS } & \multirow{2}{*}{ [178] } \\
\hline Tooth dentine & 1 & 0.70910 & 0.70910 & 0.70910 & & \\
\hline Tooth enamel & 5 & 0.70908 & 0.70945 & 0.70926 & TIMS & [179] \\
\hline
\end{tabular}

432 
As may be seen in Fig. 2, the variation of the ${ }^{87} \mathrm{Sr} /{ }^{86} \mathrm{Sr}$ within most matrices is rather small. The lowest value described in literature is for peanut, 0.695 , while the highest was 0.74985 , found in human enamel. The isotope ratios of faunal and floral samples cover a large range of values thus presenting very high amplitude of the intervals $(A=$ maximum-minimum). Both these groups are used for establishing baselines in

441 bioarchaeological studies, and therefore are extremely diverse in type, number and

442 origin of samples, which may explain such high variability. Apart from flora, human

443 enamel presents the highest amplitude in values $(A=0.045)$ which is due to the

444 elevated number of samples analysed during the last 5 years $(n=1930)$ from 51

445 different locations. Human enamel is by far the most characterized matrix in terms of 446 strontium isotope ratios.

447 Rice and hot pepper presented a very broad interval of values $(A=0.031)$, however 448 through the data available in the literature it is not possible to distinguish between the 449 two matrices and therefore reduce the amplitude of the interval. The study on olive oil 450 samples, with only 7 samples from two different provenances, registered the lowest 451 amplitude $(A=0.002)$. Nonetheless the authors were able to distinguish between 452 samples originating from Morocco and France with the developed method.

453 Amongst food matrices peanuts present the lowest and second highest ${ }^{87} \mathrm{Sr} /{ }^{86} \mathrm{Sr}$ as 454 well as the largest interval amplitude $(A=0.031)$. Despite the large number of samples 455 analysed in the study ( $n=200$ from 5 countries) this may be an indication that the 456 method employed, ICP-Q-MS, is not as precise as TIMS or MC ICP-MS, the more 457 common methods of choice.

458 Quality control is of uttermost importance in order to obtain precise and accurate 459 results. The use of clean room facilities as well as correcting for ${ }^{87} \mathrm{Rb}$ interferences 460 during analytical measurements seem to be practices well implemented in the 461 determination of strontium isotope ratios. However scarce information regarding quality 462 control data is presented and the terminology employed tends to be incoherent or 463 unclear as to the form of calculation. Terms like internal repeatability, external 464 repeatability, internal reproducibility, external reproducibility or total reproducibility 465 seem to be used in an inconsistent way by different authors, who sometimes also 466 associate the use of replicates with the evaluation of reproducibility. In addition the 467 information on the expression of uncertainty and its form of calculation (standard, 468 combined or expanded)), is often missing. Such behaviour makes it difficult to compare 469 results amongst studies, since comparability depends on the applied calibration 
470 strategies and the reported uncertainties, as stated in a recent publication dealing with

471 uncertainty calculation of isotope amount ratios [61]. With the creation of isotope

472 databases comparability amongst analytical methods, and thus amongst the data

473 produced, assumes particular importance [27]. Table 5 presents examples on the

474 information regarding sample preparation and method performance provided by some

475 of the reviewed papers. Repeatability is considered as within-run precision, while

476 between-run precision represents reproducibility [27]. Other uncertainty contributors

477 were not taken into consideration since its report in scientific papers is occasional and

478 unclear. In general papers dealing with food authentication report more information on

479 quality data when compared to the remaining applications, and therefore were selected

480 to prepare table 5.

481 As may be observed from table 5, when dealing with reference materials within-run

482 precision is quite equivalent for TIMS and MC ICP-MS. In the case of SRM 987 it is

483 generally between 0.01 and $0.02 \%$, for both methods while in food matrices is varies

484 between $0.0004 \%$ in wine and $0.3 \%$ in honey. Within-run precision is primarily

485 influenced by the nature of samples rather than the analytical technique. 
490 Table 5 Sample preparation and quality control data in food provenance studies

\begin{tabular}{|c|c|c|c|c|c|c|}
\hline \multirow{2}{*}{ Matrix } & \multirow{2}{*}{ Analytical technique } & \multicolumn{2}{|l|}{ Sample preparation } & \multirow{2}{*}{$\begin{array}{c}\text { Within run } \\
\text { precision (RSD\%) }\end{array}$} & \multirow{2}{*}{$\begin{array}{c}\text { Between run } \\
\text { precision (RSD\%) }\end{array}$} & \multirow{2}{*}{ Ref } \\
\hline & & Mineralisation & Separation of strontium from interferents & & & \\
\hline Olive oil & TIMS & $\begin{array}{l}2-100 \mathrm{~g} \text { of olive oil }+3 \mathrm{ml} \mathrm{H}_{2} \mathrm{O}_{2}+21 \mathrm{ml} \mathrm{HNO}_{3}(7 \mathrm{~N}) \text { were placed on } \\
\text { a hotplate }\left(120^{\circ} \mathrm{C}, 48 \mathrm{~h} \text { ) followed by calcination }\left(6500^{\circ} \mathrm{C} \text {, 2h). The }\right.\right. \\
\text { dry residue was dissolved with } 0.5 \mathrm{ml} \text { of } \mathrm{HNO}_{3}(2 \mathrm{M}) \text { and placed on } \\
\text { a hotplate }(5-10 \mathrm{~min})\end{array}$ & Eichrom Sr resin & $0.01 \%$ (olive oil) & $\begin{array}{l}0.001 \% \text { (SRM987) } \\
0.002-0.01 \% \text { (olive } \\
\text { oil) }\end{array}$ & [50] \\
\hline $\begin{array}{l}\text { Tomato } \\
\text { products }\end{array}$ & TIMS & $\begin{array}{l}250-300 \mathrm{mg} \text { of freeze-dried tomato products }+2-3 \mathrm{~mL} \text { of } \mathrm{HNO} 3 \\
\text { were subjected to microwave assisted digestion or chemical } \\
\text { mineralization. Samples were evaporated to dryness and re- } \\
\text { dissolved in } 10 \mathrm{~mL} \text { of } 8 \mathrm{M} \mathrm{HNO}_{3} \text {. }\end{array}$ & $\begin{array}{l}\text { Eichrom Sr resin. } 5 \mathrm{~mL} \text { of sample }+2-3 \\
\mathrm{~mL} \mathrm{HNO}\left(6-7 \mathrm{~mol} \mathrm{~L}{ }^{-1}\right) \text {. The eluate } \\
\text { containing Sr was submitted to dry } \\
\text { evaporation, recovered with } 50 \mu \mathrm{L} \text { of } \\
\mathrm{HNO}_{3} 1 \% \text { and, later on, reduced at the } \\
\text { time of the deposition on the filament. }\end{array}$ & SRM 987 & $0.006-0.05 \%$ & [77] \\
\hline $\begin{array}{l}\text { Hot pepper } \\
\text { and rice }\end{array}$ & MC ICP-MS & $\begin{array}{l}0.7 \mathrm{~g} \text { of hot pepper and rice }+ \text { concentrated } \mathrm{HNO}_{3} \text { and a } 4: 1(\mathrm{v} / \mathrm{v}) \\
\text { mixture of ultrapure concentrated } \mathrm{HNO}_{3} \text { and } \mathrm{HClO}_{4} \text { (reagent grade) } \\
\text { for complete digestion. Samples were dried in Teflon vessels and } \\
\text { re-dissolved in } 8 \mathrm{M} \mathrm{HNO}_{3} \text {. }\end{array}$ & Eichrom Sr resin & n.a & 0.001\%. (SRM987) & [70] \\
\hline Coffee Beans & MC ICP-MS & $\begin{array}{l}50 \mathrm{mg} \text { of freeze dried powdered coffee beans was digested in a } 2: 1 \\
\text { solution of } \mathrm{HNO}_{3} \text { and } \mathrm{H}_{2} \mathrm{O}_{2}\left(120^{\circ} \mathrm{C}, 2 \mathrm{~h}\right) \text {. The solution was then } \\
\text { diluted to a final volume of } 10 \mathrm{ml} \text { using Milli-Q water and stored for } \\
\text { further chemical and isotopic analysis. Approximately } 450 \mathrm{ng} \mathrm{Sr} \\
\text { from the sample solution was evaporated on a hot plate to dryness } \\
\text { at } 90^{\circ} \mathrm{C} \text { and then re-dissolved in } 0.5 \mathrm{ml} \text { of } 3 \mathrm{~N} \mathrm{HNO}_{3} \text {. }\end{array}$ & $\begin{array}{l}\text { Eichrom } \mathrm{Sr} \text { resin. The resin was washed } \\
\text { with } 3 \mathrm{ml} \text { of } 3 \mathrm{~N} \mathrm{HNO}, 3 \mathrm{ml} \text { of Milli-Q } \\
\text { water and } 5 \mathrm{ml} \text { of } 6 \mathrm{~N} \mathrm{HCl} \text { in sequence, } \\
\text { and then pre-conditioned with } 1 \mathrm{ml} \text { of } 3 \mathrm{~N} \\
\mathrm{HNO}_{3} \text {. The sample solution was loaded } \\
\text { and interferences removed with } 8 \mathrm{ml} \text { of } 3 \\
\mathrm{~N} \mathrm{HNO}_{3} \text {. The Sr fraction was eluted with } \\
4 \mathrm{ml} \text { of Milli-Q } \mathrm{Q} \text { water. }\end{array}$ & n.a. & $0.001 \%(\mathrm{SRM} 987)$ & [100] \\
\hline $\begin{array}{l}\text { Milk and } \\
\text { cheese }\end{array}$ & TIMS & $\begin{array}{l}\text { Freeze dried milk and cheese samples were dissolved in a mixture } \\
\text { of } 6 \mathrm{~N} \mathrm{HCl} \text { and } 16 \mathrm{~N} \mathrm{HNO}_{3} \text { using an Anton Paar High Pressure } \\
\text { Asher.( }\left(300{ }^{\circ} \mathrm{C}, 130 \text { bar, } 2 \mathrm{~h} \text { ). The dissolved samples were }\right. \\
\text { subsequently treated with concentrated } \mathrm{HNO}_{3} \text { and } \mathrm{H}_{2} \mathrm{O}_{2}(30 \%) \text {. The } \\
\text { samples were evaporated to dryness and re-dissolved in } 3 \mathrm{~N} \mathrm{HNO} \text {. }\end{array}$ & Eichrom Sr resin & $0.01 \%$ (cheese) & $\begin{array}{c}0.002 \%(\text { SRM987) } \\
0.001-0.03 \% \\
\text { (cheese) }\end{array}$ & [76] \\
\hline
\end{tabular}


Eichrom Sr resin SR-B100-S (50-100 Im).

High pressure digestion: $5 \mathrm{~g}$ of grape components, previously dried $\left.60{ }^{\circ} \mathrm{C}, 12 \mathrm{~h}\right)+5 \mathrm{~mL}$ of concentrated ultrapure $\mathrm{HNO}_{3}$ and $\mathrm{H}_{2} \mathrm{O}_{2}(120$ ${ }^{\circ} \mathrm{C}, 2 \mathrm{~h}$ ). The residue was then dried at $60^{\circ} \mathrm{C}$. The procedure was

Grape TIMS repeated until complete destruction of the organic matter; The

components residue was dissolved with ultrapure $2.5 \mathrm{~N} \mathrm{HCl}$ Thermal ashing: $8 \mathrm{~g}$ of grape components were dried at $60^{\circ} \mathrm{C}$ in a ultrapure $\mathrm{HCl}$ as eluent.

clean nickel crucible, and then placed in a muffle furnace, $\left(700^{\circ} \mathrm{C}\right.$, h). Ashes were then dissolved with ultrapure $25 \mathrm{NHCl}$.

\section{Microwave digestion: $5 \mathrm{~mL}$ of wine $+5 \mathrm{~mL}$ of $\mathrm{HNO}_{3}$ (room}

temperature, $2 \mathrm{~h}$ ); Step $1-9 \mathrm{~min}$, up to $60^{\circ} \mathrm{C}$; Step $2-30 \mathrm{~min}$, up to $150{ }^{\circ} \mathrm{C}$. Final volume was obtained by adding $\mathrm{HNO}_{3}$ to obtain a final solution of $\mathrm{HNO}_{3} 8 \mathrm{M}$

Low temperature procedure: $5 \mathrm{~mL}$ of wine $+5 \mathrm{~mL}$ of $\mathrm{HNO}_{3}$ (room emperature, $12 \mathrm{~h}$ ). Final volume was obtained by adding $\mathrm{HNO}_{3}$ to obtain a final solution of $\mathrm{HNO}_{3} 8 \mathrm{M}$.

Wine and grape samples $+2 \mathrm{~mL}$ of aqua regia $\left(3: 1 \mathrm{HCl}^{-} \mathrm{HNO}_{3}\right)+$ $1 \mathrm{~mL}$ of ultrapure $\mathrm{H}_{2} \mathrm{O}_{2}(24 \mathrm{~h})$. Samples were then evaporated to dryness. This sequence was repeated until complete oxidation of the organic matter was attained. $1 \mathrm{~mL}$ of $3 \mathrm{M} \mathrm{HNO}_{3}$ was added to dried samples, which were placed in an ultrasonic bath $(10 \mathrm{~min})$ and then centrifuged (10 $\mathrm{min}$ ).

$5 \mathrm{~mL}$ of wine was evaporated to dryness at $90^{\circ} \mathrm{C}$ in cleaned PFA beakers. The residue was dissolved twice in $3 \mathrm{ml}$ of ultrapure

Wine $\quad$ quality $\mathrm{H}_{2} \mathrm{O}_{2}(30 \%)\left(40^{\circ} \mathrm{C}, 1\right.$ day) and subsequently evaporated to dryness at $90^{\circ} \mathrm{C}$. The sample was then dissolved twice in $2 \mathrm{~mL}$ of suprapure concentrated $\mathrm{HNO}_{3}\left(150^{\circ} \mathrm{C}, 1\right.$ day), evaporated to dryness, and dissolved again in $1 \mathrm{~mL}$ of $3 \mathrm{~N} \mathrm{HNO}_{3}$.

$10 \mathrm{~g}$ of degassed beer was put in a cleaned Teflon vessel and heated on a hot plate until only a small amount of the sample

Beer MC ICP-MS remained. The sample was completely digested in a 6:1 mixture of

Beer MC ICP-MS remained. The sample was completely digested in a 6:1 mixture of vessels and re-dissolved in $8 \mathrm{MHNO}$ $5 \mathrm{~g}$ of dry wheat was placed in porcelain crucibles in a high-

Wheat TIMS temperature muffle furnace $\left(550-600{ }^{\circ} \mathrm{C}, 18 \mathrm{~h}\right)$. Residues were

wo times with $2 \mathrm{CV}$ ) to interferents. Sr
The resin is washed with $2 \mathrm{~mL}$ of high-

purity water and activated by $5.5 \mathrm{~mL}$ of

HNO $8 \mathrm{M} ; 5 \mathrm{~mL}$ of sample are $\mathrm{mL}$ of

and the interferences are eluted with 3.5

$\mathrm{mL}$ of $\mathrm{HNO}_{3} 8 \mathrm{M}$. The $\mathrm{Sr}$ fraction was

eluted with $7.5 \mathrm{~mL}$ of high purity water.

Sr was separated from isobar by cationexchange chromatography using Biorad AG 50W X8 resins (200-400 mesh) and

Eichrom Sr resin SR-B100-S (50-100 Im)

The resin is washed with $2 \mathrm{~mL}$ of high-

purity water and activated by $5.5 \mathrm{~mL}$ of

$\mathrm{HNO}_{3} 8 \mathrm{M} ; 5 \mathrm{~mL}$ of sample are loaded

and the interferences are eluted with 3.5

$\mathrm{mL}$ of $\mathrm{HNO}_{3} 8 \mathrm{M}$. The recovery of $\mathrm{Sr}$ is

accomplished by using $7.5 \mathrm{~mL}$ of high purity water.

\section{$0.0005-0.001 \%$}

(SRM 987)

$0.001 \%$ (soil)

$0.0004 \%$ (wine)

$0.002 \%($ SRM 987$)$

Eichrom Sr-spec resin (50-100 mesh)

$0.01-0.08 \%$

(samples)

$0.002 \%$ (SRM987)

[99]

Sr-Spec resins (100-150 $\mu \mathrm{m}$, Eichrom)

After sample loading onto the column, 14

column volumes (CV) of $3 \mathrm{~N} \mathrm{HNO}_{3}$ was

added in three steps (including rinsing

SRM987

some $13 \mathrm{CV}$ of Milli-Q water in three

steps.

Eichrom $\mathrm{Sr}$ resin. $\mathrm{Rb}$ was eluted from the

samples with $12 \mathrm{~mL}$ of $8 \mathrm{M} \mathrm{HNO}_{3}$,

followed by $16 \mathrm{~mL}$ of $1 \mathrm{M} \mathrm{HNO}_{3}$ for $\mathrm{Ca}$

and then $\mathrm{Sr}$ was collected in $8 \mathrm{~mL}$ MilliQ

water.

$0.001 \%($ SRM987)

[103]

Ion exchange chromatography column

$0.07 \%$ (wheat)

0.04\% (SRM987)

[104] 
back to the furnace. The residues were dissolved in $1 \mathrm{M} \mathrm{HNO}_{3}$.

\begin{tabular}{|c|c|c|c|c|c|c|}
\hline Honey & TIMS & $\begin{array}{l}100 \mathrm{~g} \text { of honey was placed in the furnace: Step } 1-8 \mathrm{~h} \text {, up to } 600 \\
{ }^{\circ} \mathrm{C} \text {; Step } 2-10 \mathrm{~h} \text {, at } 600{ }^{\circ} \mathrm{C} \text {. Residues were treated with } \\
\text { concentrated } \mathrm{HNO}_{3} \text { on a hot plate and then transferred back to the } \\
\text { furnace. The residues were dissolved in } \mathrm{HNO}_{3} \text {. }\end{array}$ & $\begin{array}{l}\text { lon chromatography on a Sr-specific resin } \\
\text { (Sr-spec). }\end{array}$ & $\begin{array}{l}0.2-0.3 \% \\
\text { (honey) }\end{array}$ & SRM 987 & [106] \\
\hline & TIMS & $\begin{array}{l}200 \mathrm{mg} \text { of the meat (freeze-dried or defatted dry mass) were } \\
\text { weighed into a quartz crucible and thermally ashed }\left(5000^{\circ} \mathrm{C}, 4 \mathrm{~h}\right) \text {. } \\
\text { The remaining white ash was dissolved in concentrated } \mathrm{HNO}_{3} \text {. }\end{array}$ & Sr-specific crown-ether resin (Sr-Spec $®)$ & $0.03 \%$ (beef) & $\begin{array}{l}0.004 \% \text { (SRM987) } \\
0.04-0.4 \% \text { (beef) }\end{array}$ & [93] \\
\hline Tea leaves & MC ICP-MS & $\begin{array}{l}0.25 \mathrm{~g} \text { of dried powdered tea leaves }+2 \mathrm{~mL} \mathrm{H}_{2} \mathrm{O}_{2}+6 \mathrm{~mL} \\
\text { concentrated } \mathrm{HNO}_{3} \text { Microwave digestion: step } 1-30 \mathrm{~min} \text {, up to } \\
190^{\circ} \mathrm{C} \text {; Step } 2-40 \mathrm{~min} \text {, at } 190^{\circ} \mathrm{C} \text {. Solutions were filtered through a } \\
\text { filter paper }(\mathrm{MCE} \text { no. } 42) \text { and diluted to } 12.5 \mathrm{~mL} \text { with deionised } \\
\text { water. Approximately } 150 \mathrm{ng} \text { of } \mathrm{Sr} \text { was evaporated to dryness on a } \\
\text { hot plate }\left(90^{\circ} \mathrm{C}\right) \text { and then re-dissolved in } 0.5 \mathrm{~mL} \text { of } 3 \mathrm{M} \mathrm{HNO}_{3} \text {. }\end{array}$ & $\begin{array}{l}\text { Eichrom Sr resin. The resin was pre- } \\
\text { washed with } 3 \mathrm{~mL} \text { of } 3 \mathrm{M} \mathrm{HNO}, 3 \mathrm{~mL} \\
\text { Milli-Q water and } 5 \mathrm{~mL} \text { of } 6 \mathrm{~N} \mathrm{HCl} \text { in } \\
\text { sequence and then pre-conditioned with } 1 \\
\mathrm{~mL} \text { of } 3 \mathrm{M} \mathrm{HNO}_{3} \text {. The sample solution } \\
\text { was carefully loaded onto the column, } \\
\text { where major matrix elements and } \mathrm{Rb} \\
\text { were removed by } 4 \mathrm{~mL} \text { of } 3 \mathrm{M} \mathrm{HNO} \text {. The } \\
\text { Sr fraction was eluted with } 4 \mathrm{~mL} \text { of Milli-Q } \\
\text { water. }\end{array}$ & $\begin{array}{l}0.015 \% \text { (tea } \\
\text { leaves) }\end{array}$ & $0.001 \%(\mathrm{SRM} 987)$ & [101] \\
\hline
\end{tabular}




\section{Future perspectives}

493

494 Strontium in the biological sciences has a significant number of applications, namely in

495 health sciences (diagnosis and treatment, using radioactive or stable isotopes) and as an analytical tool based on the isotopic signatures of biological samples $s$ to identify the origin of foodstuffs, diet patterns, migration habits and forensic studies along with palaeontology, archaeology and other sciences.

499 New developments concerning the application of strontium in health sciences are 500 possible, for example by using compounds of this metal associated to more efficient 501 delivery systems for diagnosis and treatment purposes.

502 It has been shown for plants [70], fish [16] and butterflies [17] that, ${ }^{87} \mathrm{Sr} /{ }^{86} \mathrm{Sr}$ does not

503 suffer fractionation from soil across trophic levels and may therefore serve as biological 504 markers for different applications. Thus, strontium isotope ratios could be inferred from 505 data on bioavailable strontium isotopes from soils, and comparing from different 506 geographical areas could give us information about evolution. Different attempts at 507 developing predictive models, isotope maps or isoscapes, have been made without 508 reaching consensus so far due to the complexity of the systems [186-189]. Although a 509 difficult task, establishing strontium isoscapes for geographical provenance of 510 foodstuffs would represent a breakthrough. Recently an archaeological bioavailable 511 strontium map of The Netherlands has been proposed [190]. Strontium isoscapes are

512 also are available for Britain [191] while in France the IRHUM database provides a data 513 set of bioavailable strontium isotope ratios [192],

514 Taking into account that strontium is mainly inert for biological species, the ratio soil

515 species and among isotopes should follow similar patterns for some closely related 516 species, even far regarding their geographical location, then these isotopes could be a 517 tool applied to the biological evolution search.

518 To analytical purposes samples of calcium biominerals replaced by strontium in low 519 amounts (e.g. teeth, bones, shells), in other biological materials (e.g. hair, fruits) and 520 derivatives (e.g. wine, cheese) have been used. However, taking into account the 521 multiple diversity of biological roles of calcium, which is the most important element in 522 types of biominerals, the employment of strontium from other biological materials, is 523 expectable.

524 On the other hand, developments in analytical techniques and instruments with high 525 measurement precision, namely MC ICP-MS and TIMS, enabled identifying relevant 526 natural fractionation in the ${ }^{88} \mathrm{Sr} /{ }^{86} \mathrm{Sr}$ ratio considered until recently to be undetectable 
527 and insignificant $[193,194]$. Souza et al. demonstrated that even though bedrock and

528 bulk soil present similar $\delta^{88 / 86} \mathrm{Sr}$ values such is not extensive to plants. In fact isotope

529 fractionation of strontium within plants was noticeable, with the $\delta^{88 / 86} \mathrm{Sr}$ values

530 decreasing while moving from roots to stems to foliar tissues [10]. This strontium

531 isotopic fractionation has been described as a temperature-dependent phenomena

532 [195].Such knowledge may lead to innovative applications in various fields like geology

$533[11,196]$, hydrology and biology $[197,198]$, in particular when combined with ${ }^{87} \mathrm{Sr} /{ }^{86} \mathrm{Sr}$..

534 Considering the natural fractionation of ${ }^{88} \mathrm{Sr} /{ }^{86} \mathrm{Sr}$ in samples, using traditional methods

535 to measure ${ }^{87} \mathrm{Sr} /{ }^{86} \mathrm{Sr}$ where the assumed constant value of ${ }^{88} \mathrm{Sr} /{ }^{86} \mathrm{Sr}$ (8.375209) is used

536 to correct for the instrument bias must be faced with caution [196].

537 Just like in ICP-MS, MC ICP-MS has the possibility of coupling to other analytical

538 techniques, such as Ablation, High Performance Liquid Chromatography or Gas

539 Chromatography opening up new possibilities with hyphenated techniques [28,121].

540 Laser ablation allows the direct analysis of solid samples thus overcoming the most

541 critical point of the analytical procedure, extraction of the strontium isotopes. This

542 technique has already been applied to different matrices as for example geological

543 materials [199] teeth and bones [121,200,201] or fish fin rays, scales and otoliths

$544[89,200]$. If in future the analytical challenges associated with LA- MC ICP-MS are

545 overcome for isotopic analysis, it would represent a significant increase in analytical

546 productivity of solid samples. On the other hand chemical speciation either via Liquid or

547 Gas Chromatography has proven its importance in trace metals analysis, particularly in

548 environmental and food analysis due to its impact in human health [202-204]. It is thus

549 expected that similar studies focusing on isotopes may lead to new advances.

550 The determination of strontium isotope ratios in biological samples like bone or tooth

551 enamel is currently very well established in laboratories. In the case of foodstuffs it has

552 already been applied to a large variety of matrices and it is expected that, with proper

553 sample preparation development, the method could be applicable to all food

554 commodities.

555 Stable isotope analysis is a field of growing interest with large amounts of data

556 generated over the last few years. Nonetheless most of these values are dispersed

557 amongst scientific publications, including preliminary studies using a reduced number

558 of samples and geographical origins (are laid out within national boundaries or more

559 rarely including two countries). It would be most useful to compile, validate and

560 compare such data on an international database making the best use of them through

561 the employment of powerful statistical tools to allow comparative studies. Elemental

562 concentrations have been widely used to develop methods for authentication of 
563 foodstuffs; however strontium isotope ratios have proved to be a more powerful tool for

564 such purpose. Nonetheless, in several cases using different techniques and crossing

565 more than one traceability marker could be valuable for a very precise tracing of the

566 geographical origin of foodstuffs, particularly in cases of similar geological environment.

567 The advantages of combining elemental concentrations and isotope ratios, both "light"

568 and "heavy" isotopes, should be a matter of interest in future research work. In the

569 specific case of processed foods or foodstuffs containing more than one ingredient,

570 and therefore with several influences on the strontium isotope ratios, this approach

571 might be particularly beneficial $[76,100,103,205]$. On the other hand, in archaeological

572 studies dealing either with past migrations, diet habits or trading activities it is common

573 to combine more than one isotopic system, in particular strontium and oxygen isotopes

$574[117,169]$. It seems evident that in these studies such approach is more beneficial

575 rather than resorting only to strontium isotopes.

\section{4. Conclusions}

577

578 The present work provides information on the application of strontium and its isotopes

579 in a diversity of biological related research fields that comprises medicine, dietary

580 habits, migration patterns, food authentication and forensic sciences. It represents a

581 valuable tool for those conducting research on such analytical applications since it

582 compiles an extensive database containing results from the last 5 years.

583 Taking into account the characteristics of strontium isotopes, as well as its chemical

584 relationships with calcium, several possible applications are available. However, care

585 must be taken to ensure proper results. Hence, according to several authors the value

586 of ${ }^{87} \mathrm{Sr} /{ }^{86} \mathrm{Sr}$ should always be found above 0.702 for all types of samples collected in

587 natural environments, since this value corresponds to the minimum value currently

588 encountered in the Earth's crust [6,12]. Lower values should be examined with caution

589 and analytical methods must be thoroughly validated.

590 The existence of an analytical quality system supporting results is essential; however

591 such control is either not implemented in analytical laboratories or it is not consistently

592 reported in recent publications. To facilitate such task the terminology employed in

593 analytical quality control should be harmonized amongst the scientific community. In

594 addition the development of matrix matching reference materials would be extremely

595 useful for future isotope ratio measurements. 
598 This project has received funding from the European Union's Horizon 2020 research

599 and innovation programme under grant agreement № 739568.

600 JALS is indebted to Fundação para a Ciência e a Tecnologia (FCT), Portugal, for

601 financial support (project UID/QUI/00100/2013).

602 The authors would like to thank José Marques for his collaboration in the design of

603 Figure 1.

\section{References}

605 [1] P. Nielsen, The biological role of strontium, Bone. 35 (2004) 583-8.

606 doi:10.1016/j.bone.2004.04.026.

607 [2] J.A. Philpotts, C.C. Schnetzler, Phenocryst-matrix partition coefficients for K, Rb, Sr and Ba, with

608

609 applications to anorthosite and basalt genesis, Geochimica et Cosmochimica Acta. 34 (1970) 307322. doi:10.1016/0016-7037(70)90108-0.

610

[3] T. Waight, Rb-Sr Geochronology (Igneous Rocks), in: Encyclopedia of Scientific Dating Methods, Springer Netherlands, Dordrecht, 2013: pp. 1-8. doi:10.1007/978-94-007-6326-5_105-1.

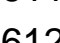

[4] United States Department of the Interior, Volcanic Rocks and Processes, in: Geological Survey

613 Research, United States Government Printing Office, Washington, 1973: pp. 141-142.

614

[5] R.C. Capo, B.W. Stewart, O.A. Chadwick, Strontium isotopes as tracers of ecosystem processes:

615 theory and methods, Geoderma. 82 (1998) 197-225. doi:10.1016/S0016-7061(97)00102-X.

616

[6] R.A. Bentley, Strontium isotopes from the earth to the archaeological skeleton: A review, Journal

617 of Archaeological Method and Theory. 13 (2006) 135-187. doi:10.1007/s10816-006-9009-x.

618

619

620

V. Höllriegl, H.Z. München, Strontium in the Environment and Possible Human Health Effects,

621

622

[8] J. Meija, T.B. Coplen, M. Berglund, W.A. Brand, P. De Bièvre, M. Gröning, et al., Isotopic Encyclopedia of Environmental Health. (2011) 268-275. doi:10.1016/B978-0-444-52272-6.006383.

624

625

626

627

628

629

630

631

632

633

634

635

636

637

638 compositions of the elements 2013 (IUPAC Technical Report), Pure and Applied Chemistry. 88 (2016). doi:10.1515/pac-2015-0503.

[9] G. Audi, F.G. Kondev, M. Wang, B. Pfeiffer, X. Sun, J. Blachot, et al., The Nubase2012 evaluation of nuclear properties, Chinese Physics C. 36 (2012) 1157-1286. doi:10.1088/16741137/36/12/001.

[10] G.F. de Souza, B.C. Reynolds, M. Kiczka, B. Bourdon, Evidence for mass-dependent isotopic fractionation of strontium in a glaciated granitic watershed, Geochimica et Cosmochimica Acta. 74 (2010) 2596-2614. doi:10.1016/j.gca.2010.02.012.

[11] H.D. Scher, E.M. Griffith, W.P. Buckley, Accuracy and precision of $88 \mathrm{Sr} / 86 \mathrm{Sr}$ and $87 \mathrm{Sr} / 86 \mathrm{Sr}$ measurements by MC-ICPMS compromised by high barium concentrations, Geochemistry, Geophysics, Geosystems. 15 (2014) 499-508. doi:10.1002/2013GC005134.

[12] M. Rosner, Geochemical and instrumental fundamentals for accurate and precise strontium isotope data of food samples: Comment on "Determination of the strontium isotope ratio by ICP. MS ginseng as a tracer of regional origin" (Choi et al., 2008), Food Chemistry. 121 (2010) 918921. doi:10.1016/j.foodchem.2010.01.019.

[13] S.A. Drivelos, C.A. Georgiou, Multi-element and multi-isotope-ratio analysis to determine the geographical origin of foods in the European Union, TrAC - Trends in Analytical Chemistry. 40 (2012) 38-51. doi:10.1016/j.trac.2012.08.003.

640

641

642

[14] A. Ranalli, P. Cabras, E. lannucci, S. Contento, Lipochromes, vitamins, aromas and other components of virgin olive oil are a $€$ ected by processing technology, Food Chemistry. 73 (2001) 445-451.

[15] A. Ranalli, A. Malfatti, L. Lucera, S. Contento, E. Sotiriou, Effects of processing techniques on the natural colourings and the other functional constituents in virgin olive oil, Food Research International. 38 (2005) 873-878. doi:10.1016/j.foodres.2005.02.011. 
[16] M. Pouilly, D. Point, F. Sondag, M. Henry, R. V. Santos, Geographical Origin of Amazonian Freshwater Fishes Fingerprinted by $87 \mathrm{Sr} / 86 \mathrm{Sr}$ Ratios on Fish Otoliths and Scales,

Environmental Science \& Technology. 48 (2014) 8980-8987. doi:10.1021/es500071w.

[17] D.T.T. Flockhart, T.K. Kyser, D. Chipley, N.G. Miller, D.R. Norris, Experimental evidence shows no fractionation of strontium isotopes ( $87 \mathrm{Sr} / 86 \mathrm{Sr}$ ) among soil, plants, and herbivores: implications for tracking wildlife and forensic science, Isotopes in Environmental and Health Studies. 51 (2015) 372-381. doi:10.1080/10256016.2015.1021345.

[18] Committee on Biomedical Isotopes Institute ofMedicine, Isotopes for Medicine and the Life Sciences, National Academy Press, Washington, D.C., D.C., 1995. http://www.nap.edu/catalog/4818.html.

[19] J. O’Neil, Stable isotope geochemistry, REVIEWS OF GEOPHYSICS AND SPACE PHYSICS. 17 (1979) 839-850.

[20] N. Walraven, B.J.H. van OS, G.T. Klaver, J.H. Baker, S.P. Vriend, Trace element concentrations and stable lead isotopes in soils as tracers of lead pollution in Graft-De Rijp, the Netherlands, Journal of Geochemical Exploration. 59 (1997) 47-58.

[21] R. Bowen, Oxygen isotopes as climatic indicators, Earth-Science Reviews. 2 (1966) 199-224. doi:10.1016/0012-8252(66)90029-8.

[22] H.P. Schwarcz, J. Melbye, M. Anne Katzenberg, M. Knyf, Stable isotopes in human skeletons of Southern Ontario: reconstructing Palaeodiet, Journal of Archaeological Science. 12 (1985) 187206. doi:10.1016/0305-4403(85)90020-2.

[23] R. van Grieken, M. de Bruin, Nomenclature for radioanalytical chemistry (IUPAC Recommendations 1994), Pure and Applied Chemistry. 66 (1994). doi:10.1351/pac199466122513.

[24] U.S. Department of Health and Human Services. Public Health Service. Agency for Toxic Substances and Disease Registry. TOXICOLOGICAL PROFILE FOR STRONTIUM, 2004.

[25] J. Aggarwal, J. Habicht-Mauche, C. Juarez, Application of heavy stable isotopes in forensic isotope geochemistry: A review, Applied Geochemistry. 23 (2008) 2658-2666. doi:10.1016/j.apgeochem.2008.05.016.

[26] B.E. Crowley, J.H. Miller, C.P. Bataille, Strontium isotopes ( $87 \mathrm{Sr} / 86 \mathrm{Sr}$ ) in terrestrial ecological and palaeoecological research: empirical efforts and recent advances in continental-scale models, Biological Reviews. (2015) n/a-n/a. doi:10.1111/brv.12217.

[27] J. Irrgeher, T. Prohaska, Application of non-traditional stable isotopes in analytical ecogeochemistry assessed by MC ICP-MS - A critical review, Analytical and Bioanalytical Chemistry. 408 (2016) 369-385. doi:10.1007/s00216-015-9025-3.

[28] P. Rodríguez-González, V.N. Epov, C. Pecheyran, D. Amouroux, O.F.X. Donard, Species-specific stable isotope analysis by the hyphenation of chromatographic techniques with MC-ICPMS, Mass Spectrometry Reviews. 31 (2012) 504-521. doi:10.1002/mas.20352.

[29] N. Vajda, C.K. Kim, Determination of radiostrontium isotopes: A review of analytical methodology, Applied Radiation and Isotopes. 68 (2010) 2306-2326. doi:10.1016/j.apradiso.2010.05.013.

684

685

686

687

688

689

690

691

[30] F. Vanhaecke, L. Balcaen, D. Malinovsky, Use of single-collector and multi-collector ICP-mass spectrometry for isotopic analysis, Journal of Analytical Atomic Spectrometry. 24 (2009) 863. doi:10.1039/b903887f.

[31] a. Gonzalvez, S. Armenta, M. de la Guardia, Trace-element composition and stable-isotope ratio for discrimination of foods with Protected Designation of Origin, TrAC - Trends in Analytical Chemistry. 28 (2009) 1295-1311. doi:10.1016/j.trac.2009.08.001.

[32] S. Kelly, K. Heaton, J. Hoogewerff, Tracing the geographical origin of food: The application of multi-element and multi-isotope analysis, Trends in Food Science and Technology. 16 (2005) 555567. doi:10.1016/j.tifs.2005.08.008.

[33] G.P. Danezis, A.S. Tsagkaris, F. Camin, V. Brusic, C.A. Georgiou, Food authentication: Techniques, trends \&amp; emerging approaches, TrAC Trends in Analytical Chemistry. (2016). doi:10.1016/j.trac.2016.02.026.

[34] M. Janin, S. Medini, I. Técher, Methods for PDO olive oils traceability: state of art and discussion about the possible contribution of strontium isotopic tool, European Food Research and Technology. (2014). doi:10.1007/s00217-014-2279-8.

[35] Y. Zhao, B. Zhang, G. Chen, A. Chen, S. Yang, Z. Ye, Recent developments in application of stable isotope analysis on agro-product authenticity and traceability, Food Chemistry. 145 (2014) 300-305. doi:10.1016/j.foodchem.2013.08.062. 
[36] C. Baffi, P.R. Trincherini, Food traceability using the $87 \mathrm{Sr} / 86 \mathrm{Sr}$ isotopic ratio mass spectrometry, European Food Research and Technology. (2016). doi:10.1007/s00217-016-2712-2.

[37] L.M. Reid, C.P. O'Donnell, G. Downey, Recent technological advances for the determination of food authenticity, Trends in Food Science and Technology. 17 (2006) 344-353. doi:10.1016/j.tifs.2006.01.006.

[38] D.M. a. M. Luykx, S. van Ruth, An overview of analytical methods for determining the geographical origin of food products, Food Chemistry. 107 (2008) 897-911. doi:10.1016/j.foodchem.2007.09.038.

[39] S. Primrose, M. Woolfe, S. Rollinson, Food forensics: Methods for determining the authenticity of foodstuffs, Trends in Food Science and Technology. 21 (2010) 582-590. doi:10.1016/j.tifs.2010.09.006

[40] L. Rock, The use of stable isotope techniques in egg authentication schemes: A review, Trends in Food Science and Technology. 28 (2012) 62-68. doi:10.1016/j.tifs.2012.04.002.

[41] Y. Oulhote, B. Le Bot, S. Deguen, P. Glorennec, Using and interpreting isotope data for source identification, TrAC Trends in Analytical Chemistry. 30 (2011) 302-312. doi:10.1016/j.trac.2010.10.015.

[42] N.R. Council, Mineral Tolerance of Animals: Second Revised Edition, (2005) 510.

[43] J. Decelle, P. Martin, K. Paborstava, D.W. Pond, G. Tarling, F. Mahé, et al., Diversity, ecology and biogeochemistry of cyst-forming acantharia (radiolaria) in the oceans, PloS One. 8 (2013) e53598. doi:10.1371/journal.pone.0053598.

[44] E. Couradeau, K. Benzerara, E. Gérard, D. Moreira, S. Bernard, G.E. Brown, et al., An earlybranching microbialite cyanobacterium forms intracellular carbonates, Science (New York, N.Y.). 336 (2012) 459-62. doi:10.1126/science.1216171.

[45] L. Zhao, B.R. Schöne, R. Mertz-Kraus, Controls on strontium and barium incorporation into freshwater bivalve shells (Corbicula fluminea), Palaeogeography, Palaeoclimatology, Palaeoecology. 465 (2017) 386-394. doi:10.1016/j.palaeo.2015.11.040.

[46] Infoplease, Periodic table, (n.d.). http://www.infoplease.com/periodictable.php.

[47] Wikipedia the free encyclopedia, Abundance of elements in Earth's crust, (2015). https://en.wikipedia.org/wiki/Abundance_of_elements_in_Earth\%27s_crust (accessed July 1, 2015).

[48] H. Sverdrup, M. Johnson, R. Fleming, Chemistry of Sea Water, in: The Oceans Their Physics, Chemistry, and General Biology, Prentice-Hall, Inc., 1942: pp. 165-227.

http://scholar.google.com/scholar?hl=en\&btnG=Search\&q=intitle:19+42.+The+oceans:+their+physi cs,+chemistry+and+general+biology\#0.

[49] S. Millour, L. Noël, R. Chekri, C. Vastel, A. Kadar, V. Sirot, et al., Strontium, silver, tin, iron, tellurium, gallium, germanium, barium and vanadium levels in foodstuffs from the Second French Total Diet Study, Journal of Food Composition and Analysis. 25 (2012) 108-129. doi:10.1016/j.jfca.2011.10.004.

[50] S. Medini, M. Janin, P. Verdoux, I. Techer, Methodological development for $87 \mathrm{Sr} / 86 \mathrm{Sr}$ measurement in olive oil and preliminary discussion of its use for geographical traceability of PDO Nîmes (France), Food Chemistry. 171 (2015) 78-83. doi:10.1016/j.foodchem.2014.08.121.

[51] B.J. Tipple, T. Chau, L.A. Chesson, D.P. Fernandez, J.R. Ehleringer, Isolation of strontium pools and isotope ratios in modern human hair, Analytica Chimica Acta. 798 (2013) 64-73. doi:10.1016/j.aca.2013.08.054.

[52] G. Vautour, A. Poirier, D. Widory, Tracking mobility using human hair: What can we learn from lead and strontium isotopes?, Science \& Justice. 55 (2015) 63-71. doi:10.1016/j.scijus.2014.10.001.

[53] B. Valentine, G.D. Kamenov, J.M. Kenoyer, V. Shinde, V. Mushrif-Tripathy, E. Otarola-Castillo, et al., Evidence for Patterns of Selective Urban Migration in the Greater Indus Valley (2600-1900 BC): A Lead and Strontium Isotope Mortuary Analysis, PLOS ONE. 10 (2015) e0123103. doi:10.1371/journal.pone.0123103.

[54] A.C. Ross, J.E. Manson, S.A. Abrams, J.F. Aloia, P.M. Brannon, S.K. Clinton, et al., The 2011 Report on Dietary Reference Intakes for Calcium and Vitamin D from the Institute of Medicine: What Clinicians Need to Know, The Journal of Clinical Endocrinology \& Metabolism. 96 (2011) 5358. doi:10.1210/jc.2010-2704. means of inductively coupled plasma-mass spectrometry for provenancing purposes - A review, 

doi:10.1016/j.sab.2010.06.005.

[56] L. Yang, Accurate and precise determination of isotopic ratios by MC-ICP-MS: a review., Mass Spectrometry Reviews. 28 (2009) 990-1011. doi:10.1002/mas.20251.

[57] E.M. Krupp, C. Pécheyran, H. Pinaly, M. Motelica-Heino, D. Koller, S.M.M. Young, et al., Isotopic precision for a lead species (PbEt4) using capillary gas chromatography coupled to inductively coupled plasma-multicollector mass spectrometry, Spectrochimica Acta Part B: Atomic Spectroscopy. 56 (2001) 1233-1240. doi:10.1016/S0584-8547(01)00204-X.

[58] C.-K. Jeong, H. Jang, G.-J. Lee, Study on strontium isotope abundance-ratio measurements by using a 13-MeV proton beam, Journal of the Korean Physical Society. 69 (2016) 1121-1124. doi:10.3938/jkps.69.1121.

[59] T. Stephan, R. Trappitsch, A.M. Davis, M.J. Pellin, D. Rost, M.R. Savina, et al., CHILI - the Chicago Instrument for Laser lonization - a new tool for isotope measurements in cosmochemistry, International Journal of Mass Spectrometry. 407 (2016) 1-15. doi:10.1016/j.jims.2016.06.001.

[60] J. Irrgeher, A. Zitek, M. Cervicek, T. Prohaska, Analytical factors to be considered for the application of enriched strontium spikes to monitor biological systems, J. Anal. At. Spectrom. 29 (2014) 193-200. doi:10.1039/C3JA50212K

[61] M. Horsky, J. Irrgeher, T. Prohaska, Evaluation strategies and uncertainty calculation of isotope amount ratios measured by MC ICP-MS on the example of Sr, Analytical and Bioanalytical Chemistry. 408 (2016) 351-367. doi:10.1007/s00216-015-9003-9.

[62] B.L.A. Charlier, G.M. Nowell, I.J. Parkinson, S.P. Kelley, D.G. Pearson, K.W. Burton, High temperature strontium stable isotope behaviour in the early solar system and planetary bodies, Earth and Planetary Science Letters. 329-330 (2012) 31-40. doi:10.1016/j.epsl.2012.02.008.

[63] T. Kleine, Radiogenic Isotopes, in: Encyclopedia of Astrobiology, Springer Berlin Heidelberg, Berlin, Heidelberg, 2011: pp. 1424-1430. doi:10.1007/978-3-642-11274-4_1343.

[64] A. V. Dunin, N.A. Nerozin, N.R. Togaeva, S. V. Khamyanov, V. V. Shapovalov, Extraction of Sr-82, Raw Material for Radiopharmaceutical Production, Pharmaceutical Chemistry Journal. 48 (2014) 395-397. doi:10.1007/s11094-014-1118-3.

[65] I. Kuroda, Effective use of strontium-89 in osseous metastases, Annals of Nuclear Medicine. 26 (2012) 197-206. doi:10.1007/s12149-011-0560-5.

[66] X. jiao Qin, H. mei Chen, L. Guo, Y. yuan Guo, Low-dose strontium-90 irradiation is effective in preventing the recurrence of pterygia: A ten-year study, PLOS ONE. 7 (2012). doi:10.1371/journal.pone.0043500.

[67] J.- Reginster, Strontium Ranelate in Osteoporosis, Current Pharmaceutical Design. 8 (2002) 1907-1916. doi:10.2174/1381612023393639.

[68] European Medicines Agency, Protelos and Osseor Article-20 procedure - Protelos/Osseor to remain available but with further restrictions, 2014.

http://www.ema.europa.eu/docs/en_GB/document_library/Referrals_document/Protelos_and_Oss eor/European_Commission_final_decision/WC500173034.pdf.

[69] J.-Y. Reginster, C. Beaudart, A. Neuprez, O. Bruyère, Strontium ranelate in the treatment of knee osteoarthritis: new insights and emerging clinical evidence., Therapeutic Advances in Musculoskeletal Disease. 5 (2013) 268-276. doi:10.1177/1759720X13500862.

[70] B.-Y. Song, J.-S. Ryu, H.S. Shin, K.-S. Lee, Determination of the Source of Bioavailable Sr Using $87 \mathrm{Sr} / 86$ Sr Tracers: A Case Study of Hot Pepper and Rice, Journal of Agricultural and Food Chemistry. 62 (2014) 9232-9238. doi:10.1021/jf503498r.

[71] B.-Y. Song, M.K. Gautam, J.-S. Ryu, D. Lee, K.-S. Lee, Effects of bedrock on the chemical and Sr isotopic compositions of plants, Environmental Earth Sciences. 74 (2015) 829-837. doi:10.1007/s12665-015-4087-2.

[72] C. Durante, C. Baschieri, L. Bertacchini, M. Cocchi, S. Sighinolfi, M. Silvestri, et al., Geographical traceability based on $87 \mathrm{Sr} / 86 \mathrm{Sr}$ indicator: A first approach for PDO Lambrusco wines from Modena, Food Chemistry. 141 (2013) 2779-87. doi:10.1016/j.foodchem.2013.05.108.

[73] C. Durante, C. Baschieri, L. Bertacchini, D. Bertelli, M. Cocchi, A. Marchetti, et al., An analytical approach to $\mathrm{Sr}$ isotope ratio determination in Lambrusco wines for geographical traceability purposes, Food Chemistry. 173 (2015) 557-63. doi:10.1016/j.foodchem.2014.10.086. 
[75] International olive council, World Olive Oil Figures - International Olive Council, (n.d.). http://www.internationaloliveoil.org/estaticos/view/131-world-olive-oil-figures (accessed January 7 , 2015).

[76] R. Stevenson, S. Desrochers, J.-F. Hélie, Stable and radiogenic isotopes as indicators of agri-food provenance: Insights from artisanal cheeses from Quebec, Canada, International Dairy Journal. (2015). doi:10.1016/j.idairyj.2015.04.003.

[77] P.R. Trincherini, C. Baffi, P. Barbero, E. Pizzoglio, S. Spalla, Precise determination of strontium isotope ratios by TIMS to authenticate tomato geographical origin, Food Chemistry. 145 (2014) 349-355. doi:10.1016/j.foodchem.2013.08.030.

[78] Y. Zhu, A. Hioki, K. Chiba, Measurement of strontium isotope ratio in nitric acid extract of peanut testa by ICP-Q-MS after removal of Rb by extraction with pure water, Talanta. 119 (2014) 596600. doi:10.1016/j.talanta.2013.11.058.

[79] K. G. Heumann, S. M. Gallus, G. Rädlinger, J. Vogl, Precision and accuracy in isotope ratio measurements by plasma source mass spectrometry, Journal of Analytical Atomic Spectrometry. 13 (1998) 1001. doi:10.1039/a801965g.

833

834

835

836

[80] B.A. Wolff, B.M. Johnson, C.M. Landress, B. Gillanders, Classification of hatchery and wild fish using natural geochemical signatures in otoliths, fin rays, and scales of an endangered catostomid, Canadian Journal of Fisheries and Aquatic Sciences. 70 (2013) 1775-1784. doi:10.1139/cjfas2013-0116.

837

838

839

840

841

842

843

844

[81] J. Martin, G. Bareille, S. Berail, C. Pecheyran, F. Daverat, N. Bru, et al., Spatial and temporal variations in otolith chemistry and relationships with water chemistry: a useful tool to distinguish Atlantic salmon Salmo salar parr from different natal streams, Journal of Fish Biology. 82 (2013) 1556-1581. doi:10.1111/jfb.12089.

[82] J. Martin, G. Bareille, S. Berail, C. Pécheyran, F. Gueraud, F. Lange, et al., Persistence of a southern Atlantic salmon population: diversity of natal origins from otolith elemental and Sr isotopic signatures, Canadian Journal of Fisheries and Aquatic Sciences. 70 (2013) 182-197. doi:10.1139/cjfas-2012-0284.

845

[83] S.R. Brennan, D.P. Fernandez, C.E. Zimmerman, T.E. Cerling, R.J. Brown, M.J. Wooller, Strontium isotopes in otoliths of a non-migratory fish (slimy sculpin): Implications for provenance studies, Geochimica et Cosmochimica Acta. 149 (2015) 32-45. doi:10.1016/j.gca.2014.10.032.

848

849

[84] C.C. Muhlfeld, S.R. Thorrold, T.E. McMahon, B. Marotz, B. Gillanders, Estimating westslope cutthroat trout ( Oncorhynchus clarkii lewisi) movements in a river network using strontium isoscapes, Canadian Journal of Fisheries and Aquatic Sciences. 69 (2012) 906-915. doi:10.1139/£2012-033.

[85] J.I. Macdonald, D.G. McNeil, D.A. Crook, Asteriscus v. lapillus: comparing the chemistry of two otolith types and their ability to delineate riverine populations of common carp Cyprinus carpio, Journal of Fish Biology. 81 (2012) 1715-1729. doi:10.1111/j.1095-8649.2012.03443.x.

855

[86] B.A. Wolff, B.M. Johnson, A.R. Breton, P.J. Martinez, D.L. Winkelman, B. Gillanders, Origins of invasive piscivores determined from the strontium isotope ratio ( $87 \mathrm{Sr} / 86 \mathrm{Sr}$ ) of otoliths, Canadian Journal of Fisheries and Aquatic Sciences. 69 (2012) 724-739. doi:10.1139/f2012-009.

[87] A.J. Padilla, R.J. Brown, M.J. Wooller, Determining the Movements and Distribution of Anadromous Bering Ciscoes by Use of Otolith Strontium Isotopes, Transactions of the American Fisheries Society. 145 (2016) 1374-1385. doi:10.1080/00028487.2016.1225599.

861

[88] R. Johnson, J. Garza, R. MacFarlane, C. Grimes, C. Phillis, P. Koch, et al., Isotopes and genes reveal freshwater origins of Chinook salmon Oncorhynchus tshawytscha aggregations in California's coastal ocean, Marine Ecology Progress Series. 548 (2016) 181-196. doi:10.3354/meps11623.

865

[89] M. Willmes, J.J.G. Glessner, S.A. Carleton, P.C. Gerrity, J.A. Hobbs, $87 \mathrm{Sr} / 86 \mathrm{Sr}$ isotope ratio analysis by laser ablation MC-ICP-MS in scales, spines, and fin rays as a nonlethal alternative to otoliths for reconstructing fish life history, Canadian Journal of Fisheries and Aquatic Sciences. 73 (2016) 1852-1860. doi:10.1139/cjfas-2016-0103.

869

870

871

872

873

[90] Y.-S. Bong, W.-J. Shin, M.K. Gautam, Y.-J. Jeong, A.-R. Lee, C.-S. Jang, et al., Determining the geographical origin of Chinese cabbages using multielement composition and strontium isotope ratio analyses, Food Chemistry. 135 (2012) 2666-2674. doi:10.1016/j.foodchem.2012.07.045.

[91] Y.-S. BONG, M.K. GAUTAM, M.-R. LA, K.-S. LEE, Geographic Origins of Korean and Chinese Kimchi Determined by Multiple Elements, Bioscience, Biotechnology, and Biochemistry. 76 (2012) 
2096-2100. doi:10.1271/bbb.120471.

[92] K. Ariyama, M. Shinozaki, A. Kawasaki, Determination of the Geographic Origin of Rice by Chemometrics with Strontium and Lead Isotope Ratios and Multielement Concentrations, Journal of Agricultural and Food Chemistry. 60 (2012) 1628-1634. doi:10.1021/j204296p.

[93] S. Rummel, C.H. Dekant, S. Hölzl, S.D. Kelly, M. Baxter, N. Marigheto, et al., Sr isotope measurements in beef-analytical challenge and first results, Analytical and Bioanalytical Chemistry. 402 (2012) 2837-2848. doi:10.1007/s00216-012-5759-3.

[94] H. HIRAOKA, S. MORITA, A. IZAWA, K. AOYAMA, K.-C. SHIN, T. NAKANO, Tracing the Geographical Origin of Onions by Strontium Isotope Ratio and Strontium Content, Analytical Sciences. 32 (2016) 781-788. doi:10.2116/analsci.32.781.

[95] R. Petrini, L. Sansone, F.F. Slejko, A. Buccianti, P. Marcuzzo, D. Tomasi, The (87)Sr/(86)Sr strontium isotopic systematics applied to Glera vineyards: a tracer for the geographical origin of the Prosecco, Food Chemistry. 170 (2015) 138-44. doi:10.1016/j.foodchem.2014.08.051.

[96] J.R. Fernandes, L. Pereira, P. Jorge, L. Moreira, H. Gonçalves, L. Coelho, et al., Wine fingerprinting using a bio-geochemical approach, BIO Web of Conferences. 5 (2015) 2021. doi:10.1051/bioconf/20150502021.

[97] S. Marchionni, A. Buccianti, A. Bollati, E. Braschi, F. Cifelli, P. Molin, et al., Conservation of $87 \mathrm{Sr} / 86 \mathrm{Sr}$ isotopic ratios during the winemaking processes of "Red" wines to validate their use as geographic tracer, Food Chemistry. 190 (2016) 777-785. doi:10.1016/j.foodchem.2015.06.026.

893

894

[98] S. Catarino, C. Moreira, A. Kaya, R.B. de Sousa, A. Curvelo-Garcia, M. de Pinho, et al., Effect of new and conventional technological processes on the terroir marker $87 \mathrm{Sr} / 86 \mathrm{Sr}$, BIO Web of Conferences. 7 (2016) 2003. doi:10.1051/bioconf/20160702003.

896

897

[99] V. Vinciguerra, R. Stevenson, K. Pedneault, A. Poirier, J.-F. Hélie, D. Widory, Strontium isotope characterization of wines from Quebec, Canada, Food Chemistry. 210 (2016) 121-128. doi:10.1016/j.foodchem.2016.04.017.

[100] H.-C. Liu, C.-F. You, C.-Y. Chen, Y.-C. Liu, M.-T. Chung, Geographic determination of coffee beans using multi-element analysis and isotope ratios of boron and strontium, Food Chemistry. 142 (2014) 439-45. doi:10.1016/j.foodchem.2013.07.082.

[101] C.-T. Chang, C.-F. You, S.K. Aggarwal, C.-H. Chung, H.-C. Chao, H.-C. Liu, Boron and strontium isotope ratios and major/trace elements concentrations in tea leaves at four major tea growing gardens in Taiwan, Environmental Geochemistry and Health. 38 (2016) 737-748. doi:10.1007/s10653-015-9757-1.

[102] G. Rees, S.D. Kelly, P. Cairns, H. Ueckermann, S. Hoelzl, A. Rossmann, et al., Verifying the geographical origin of poultry: The application of Stable Isotope and Trace Element (SITE) Analysis, Food Control. (2016). doi:10.1016/j.foodcont.2016.02.018.

[103] Y.-S. Bong, J.-S. Ryu, S.-H. Choi, M.-R. La, K.-S. Lee, Investigation of the geographical provenance of the beer available in South Korea using multielements and isotopes, Food Control. 60 (2016) 378-381. doi:10.1016/j.foodcont.2015.08.017.

912

913

914

915

916

917

918

[104] N.S. Podio, M. V. Baroni, R.G. Badini, M. Inga, H.A. Ostera, M. Cagnoni, et al., Elemental and Isotopic Fingerprint of Argentinean Wheat. Matching Soil, Water, and Crop Composition to Differentiate Provenance, Journal of Agricultural and Food Chemistry. 61 (2013) 3763-3773. doi:10.1021/jf305258r.

[105] H. Liu, Y. Wei, H. Lu, S. Wei, T. Jiang, Y. Zhang, et al., Combination of the $87 \mathrm{Sr} / 86 \mathrm{Sr}$ ratio and light stable isotopic values $(\delta 13 \mathrm{C}, \delta 15 \mathrm{~N}$ and $\delta \mathrm{D})$ for identifying the geographical origin of winter wheat in China, Food Chemistry. 212 (2016) 367-373. doi:10.1016/j.foodchem.2016.06.002.

[106] M. V. Baroni, N.S. Podio, R.G. Badini, M. Inga, H.A. Ostera, M. Cagnoni, et al., Linking Soil, Water, and Honey Composition To Assess the Geographical Origin of Argentinean Honey by Multielemental and Isotopic Analyses, Journal of Agricultural and Food Chemistry. 63 (2015) 4638-4645. doi:10.1021/j55060112.

[107] C.A. Makarewicz, J. Sealy, Dietary reconstruction, mobility, and the analysis of ancient skeletal tissues: Expanding the prospects of stable isotope research in archaeology, Journal of Archaeological Science. 56 (2015) 146-158. doi:10.1016/j.jas.2015.02.035.

[108] P.A. Slater, K.M. Hedman, T.E. Emerson, Immigrants at the Mississippian polity of Cahokia: Strontium isotope evidence for population movement, Journal of Archaeological Science. 44 (2014) 117-127. doi:10.1016/j.jas.2014.01.022.

[109] A.J. Waterman, D.W. Peate, A.M. Silva, J.T. Thomas, In search of homelands: Using strontium isotopes to identify biological markers of mobility in late prehistoric Portugal, Journal of 
[110] D.T. Price, S. Nakamura, S. Suzuki, J.H. Burton, V. Tiesler, New isotope data on Maya mobility and enclaves at Classic Copan, Honduras, Journal of Anthropological Archaeology. 36 (2014) 32 47. doi:10.1016/j.jaa.2014.02.003.

[111] J. Pokines, S. Symes, Manual of Forensic Taphonomy, 2013.

[112] M. Sponheimer, J.A. Lee-Thorp, Enamel diagenesis at South African Australopith sites: Implications for paleoecological reconstruction with trace elements, Geochimica et Cosmochimica Acta. 70 (2006) 1644-1654. doi:10.1016/j.gca.2005.12.022.

[113] P. Budda, J. Montgomery, B. Barreiro, R.G. Thomas, Differential diagenesis of strontium in archaeological human dental tissues, Applied Geochemistry. 15 (2000) 687-694.

[114] C. Minniti, S. Valenzuela-Lamas, J. Evans, U. Albarella, Widening the market. Strontium isotope analysis on cattle teeth from Owslebury (Hampshire, UK) highlights changes in livestock supply between the Iron Age and the Roman period, Journal of Archaeological Science. 42 (2014) 305314. doi:10.1016/j.jas.2013.10.008.

[115] L.A. Gregoricka, Residential mobility and social identity in the periphery: strontium isotope analysis of archaeological tooth enamel from southeastern Arabia, Journal of Archaeological Science. 40 (2013) 452-464. doi:10.1016/j.jas.2012.07.017.

[116] J.E. Laffoon, G.R. Davies, M.L.P. Hoogland, C.L. Hofman, Spatial variation of biologically available strontium isotopes (87Sr/86Sr) in an archipelagic setting: a case study from the Caribbean, Journal of Archaeological Science. 39 (2012) 2371-2384. doi:10.1016/j.jas.2012.02.002.

[117] E. McManus, J. Montgomery, J. Evans, A. Lamb, R. Brettell, J. Jelsma, "To the Land or to the Sea": Diet and Mobility in Early Medieval Frisia, The Journal of Island and Coastal Archaeology. 8 (2013) 255-277. doi:10.1080/15564894.2013.787565.

[118] P. Degryse, D. De Muynck, S. Delporte, S. Boyen, L. Jadoul, J. De Winne, et al., Strontium isotopic analysis as an experimental auxiliary technique in forensic identification of human remains, Analytical Methods. 4 (2012) 2674. doi:10.1039/c2ay25035g.

[119] A.-F. Maurer, S.J.G. Galer, C. Knipper, L. Beierlein, E. V. Nunn, D. Peters, et al., Bioavailable $87 \mathrm{Sr} / 86 \mathrm{Sr}$ in different environmental samples - Effects of anthropogenic contamination and implications for isoscapes in past migration studies, Science of The Total Environment. 433 (2012) 216-229. doi:10.1016/j.scitotenv.2012.06.046.

[120] H. Wilhelmson, T. Ahlström, Iron Age migration on the island of Öland: Apportionment of strontium by means of Bayesian mixing analysis, Journal of Archaeological Science. 64 (2015) 30-45. doi:10.1016/j.jas.2015.09.007.

[121] P.J. Le Roux, J. a. Lee-Thorp, S.R. Copeland, M. Sponheimer, D.J. de Ruiter, Strontium isotope analysis of curved tooth enamel surfaces by laser-ablation multi-collector ICP-MS, Palaeogeography, Palaeoclimatology, Palaeoecology. (2014). doi:10.1016/j.palaeo.2014.09.007.

[122] B.L. Turner, M.K. Zuckerman, E.M. Garofalo, A. Wilson, G.D. Kamenov, D.R. Hunt, et al., Diet and death in times of war: isotopic and osteological analysis of mummified human remains from southern Mongolia, Journal of Archaeological Science. 39 (2012) 3125-3140. doi:10.1016/j.jas.2012.04.053.

[123] L.E. Wright, Immigration to Tikal, Guatemala: Evidence from stable strontium and oxygen isotopes, Journal of Anthropological Archaeology. 31 (2012) 334-352. doi:10.1016/j.jaa.2012.02.001.

[124] K.A. Hemer, J.A. Evans, C.A. Chenery, A.L. Lamb, No Man is an island: evidence of pre-Viking Age migration to the Isle of Man, Journal of Archaeological Science. 52 (2014) 242-249. doi:10.1016/j.jas.2014.08.031.

[125] E.J. Kendall, J. Montgomery, J.A. Evans, C. Stantis, V. Mueller, Mobility, mortality, and the middle ages: Identification of migrant individuals in a 14th century black death cemetery population, American Journal of Physical Anthropology. 150 (2013) 210-222. doi:10.1002/ajpa.22194.

[126] K.A. Hemer, J.A. Evans, C.A. Chenery, A.L. Lamb, Evidence of early medieval trade and migration between Wales and the Mediterranean Sea region, Journal of Archaeological Science. 40 (2013) 2352-2359. doi:10.1016/j.jas.2013.01.014.

[127] R. Kinaston, S. Bedford, M. Richards, S. Hawkins, A. Gray, K. Jaouen, et al., Diet and Human Mobility from the Lapita to the Early Historic Period on Uripiv Island, Northeast Malakula, Vanuatu, PLoS ONE. 9 (2014) e104071. doi:10.1371/journal.pone.0104071. 
[129] A.D. Somerville, M.J. Schoeninger, G.E. Braswell, Political alliance, residential mobility, and diet at the ancient Maya city of Pusilha, Belize, Journal of Anthropological Archaeology. 41 (2016) 147158. doi:10.1016/j.jaa.2015.11.004.

[130] B. Chase, D. Meiggs, P. Ajithprasad, P.A. Slater, Pastoral land-use of the Indus Civilization in Gujarat: faunal analyses and biogenic isotopes at Bagasra, Journal of Archaeological Science. 50 (2014) 1-15. doi:10.1016/j.jas.2014.06.013.

[131] H. Shaw, J. Montgomery, R. Redfern, R. Gowland, J. Evans, Identifying migrants in Roman London using lead and strontium stable isotopes, Journal of Archaeological Science. 66 (2016) 57-68. doi:10.1016/j.jas.2015.12.001.

[132] C. Knipper, M. Fragata, N. Nicklisch, A. Siebert, A. Szécsényi-Nagy, V. Hubensack, et al., A distinct section of the early bronze age society? Stable isotope investigations of burials in settlement pits and multiple inhumations of the Únětice culture in central germany, American Journal of Physical Anthropology. 159 (2016) 496-516. doi:10.1002/ajpa.22892.

[133] K. Killgrove, J. Montgomery, All Roads Lead to Rome: Exploring Human Migration to the Eternal City through Biochemistry of Skeletons from Two Imperial-Era Cemeteries (1st-3rd c AD), PLOS ONE. 11 (2016) e0147585. doi:10.1371/journal.pone.0147585.

[134] A.F. Carvalho, F. Alves-Cardoso, D. Gonçalves, R. Granja, J.L. Cardoso, R.M. Dean, et al., The Bom Santo Cave (Lisbon, Portugal): Catchment, Diet, and Patterns of Mobility of a Middle Neolithic Population, European Journal of Archaeology. 19 (2016) 187-214. doi:10.1179/1461957115Y.0000000014.

[135] D.S. Kurin, E.M. Lofaro, D.E. Gómez Choque, J. Krigbaum, A Bioarchaeological and Biogeochemical Study of Warfare and Mobility in Andahuaylas, Peru (ca. $<$ scp $>$ ad $</$ scp $>1160$ 1260), International Journal of Osteoarchaeology. 26 (2016) 93-103. doi:10.1002/oa.2398.

[136] M.Q.R. Bastos, R. V. Santos, S.M.F. M. de Souza, C. Rodrigues-Carvalho, R.H. Tykot, D.C. Cook, et al., Isotopic study of geographic origins and diet of enslaved Africans buried in two Brazilian cemeteries, Journal of Archaeological Science. 70 (2016) 82-90. doi:10.1016/j.jas.2016.04.020.

[137] S. Neil, J. Evans, J. Montgomery, C. Scarre, Isotopic evidence for residential mobility of farming communities during the transition to agriculture in Britain, Royal Society Open Science. 3 (2016) 150522. doi:10.1098/rsos.150522.

[138] E. Oras, V. Lang, E. Rannamäe, L. Varul, M. Konsa, J. Limbo-Simovart, et al., TRACING PREHISTORIC MIGRATION: ISOTOPE ANALYSIS OF BRONZE AND PRE-ROMAN IRON AGE COASTAL BURIALS IN ESTONIA, Estonian Journal of Archaeology. 20 (2016) 3. doi:10.3176/arch.2016.1.01.

[139] A.L. Lamb, J.E. Evans, R. Buckley, J. Appleby, Multi-isotope analysis demonstrates significant lifestyle changes in King Richard III, Journal of Archaeological Science. 50 (2014) 559-565. doi:10.1016/j.jas.2014.06.021.

[140] G. Goude, F. Castorina, E. Herrscher, S. Cabut, M.A. Tafuri, First Strontium Isotope Evidence of Mobility in the Neolithic of Southern France, European Journal of Archaeology. 15 (2012) $421-$ 439. doi:10.1179/1461957112Y.0000000017.

[141] S. Kusaka, T. Nakano, W. Morita, M. Nakatsukasa, Strontium isotope analysis to reveal migration in relation to climate change and ritual tooth ablation of Jomon skeletal remains from western Japan, Journal of Anthropological Archaeology. 31 (2012) 551-563. doi:10.1016/j.jaa.2012.05.004.

[142] K.J. Knudson, B. O'Donnabhain, C. Carver, R. Cleland, T.D. Price, Migration and Viking Dublin: paleomobility and paleodiet through isotopic analyses, Journal of Archaeological Science. 39 (2012) 308-320. doi:10.1016/j.jas.2011.09.014.

[143] M.R. Buzon, C.A. Conlee, A. Simonetti, G.J. Bowen, The consequences of Wari contact in the Nasca region during the Middle Horizon: archaeological, skeletal, and isotopic evidence, Journal of Archaeological Science. 39 (2012) 2627-2636. doi:10.1016/j.jas.2012.04.003.

[144] K.J. Knudson, W.J. Pestle, C. Torres-Rouff, G. Pimentel, Assessing the life history of an andean traveller through biogeochemistry: Stable and radiogenic isotope analyses of archaeological human remains from Northern Chile, International Journal of Osteoarchaeology. 22 (2012) 435451. doi:10.1002/oa.1217.

[145] J.I. Giblin, K.J. Knudson, Z. Bereczki, G. Pálfi, I. Pap, Strontium isotope analysis and human mobility during the Neolithic and Copper Age: a case study from the Great Hungarian Plain, Journal of Archaeological Science. 40 (2013) 227-239. doi:10.1016/j.jas.2012.08.024.

[146] K.L. Nado, S.J. Marsteller, L.M. King, B.M. Daverman, C. Torres-Rouff, K.J. Knudson, EXAMINING LOCAL SOCIAL IDENTITIES THROUGH PATTERNS OF BIOLOGICAL AND 
CULTURAL VARIATION IN THE SOLCOR AYLLU, SAN PEDRO DE ATACAMA, CHILE, Chungará (Arica). 44 (2012) 341-357. doi:10.4067/S0717-73562012000200010.

[147] C. Lang, J. Peters, N. Pöllath, K. Schmidt, G. Grupe, Gazelle behaviour and human presence at early Neolithic Göbekli Tepe, south-east Anatolia, World Archaeology. 45 (2013) 410-429. doi:10.1080/00438243.2013.820648.

[148] X. Zhang, J. Burton, Z. Jin, M. Xiao, A. Fan, J. Xu, Isotope studies of human remains from Mayutian, Yunnan Province, China, Journal of Archaeological Science. 50 (2014) 414-419. doi:10.1016/j.jas.2014.08.001.

[149] K.W. Alt, C. Knipper, D. Peters, W. Müller, A.-F. Maurer, I. Kollig, et al., Lombards on the Move An Integrative Study of the Migration Period Cemetery at Szólád, Hungary, PLoS ONE. 9 (2014) e110793. doi:10.1371/journal.pone.0110793.

[150] J.W. Eerkens, G.H. Barfod, G.A. Jorgenson, C. Peske, Tracing the mobility of individuals using stable isotope signatures in biological tissues: "locals" and "non-locals" in an ancient case of violent death from Central California, Journal of Archaeological Science. 41 (2014) 474-481. doi:10.1016/j.jas.2013.09.014.

[151] Y. Bäckström, T.D. Price, Social identity and mobility at a pre-industrial mining complex, Sweden, Journal of Archaeological Science. 66 (2016) 154-168. doi:10.1016/j.jas.2016.01.004.

[152] K.W. Alt, S. Zesch, R. Garrido-Pena, C. Knipper, A. Szécsényi-Nagy, C. Roth, et al., A Community in Life and Death: The Late Neolithic Megalithic Tomb at Alto de Reinoso (Burgos, Spain), PLOS ONE. 11 (2016) e0146176. doi:10.1371/journal.pone.0146176.

[153] C. Snoeck, J. Pouncett, G. Ramsey, I.G. Meighan, N. Mattielli, S. Goderis, et al., Mobility during the neolithic and bronze age in northern ireland explored using strontium isotope analysis of cremated human bone, American Journal of Physical Anthropology. 160 (2016) 397-413. doi:10.1002/ajpa.22977.

[154] M.A. Tafuri, P.D. Fullagar, T.C. O'Connell, M.G. Belcastro, P. lacumin, C. Conati Barbaro, et al., Life and Death in Neolithic Southeastern Italy: The Strontium Isotopic Evidence, International Journal of Osteoarchaeology. 26 (2016) 1045-1057. doi:10.1002/oa.2516.

[155] G. HUELGA-SUAREZ, S. DIEZ-FERNÁNDEZ, M. MOLDOVAN, A.F. PARDIÑAS, B. LÓPEZ, J.I. GARCÍA ALONSO, The quest for the soldier's rest: combining anthropological and archaeochemical approaches to study social and occupational diversity in the medieval graveyard of San Andrés de Arroyo (Palencia, Spain), Anthropological Science. 124 (2016) 169-184. doi:10.1537/ase.161005.

[156] X. Wang, Z. Tang, J. Wu, X. Wu, Y. Wu, X. Zhou, Strontium isotope evidence for a highly mobile population on the Pamir Plateau 2500 years ago, Scientific Reports. 6 (2016) 35162. doi:10.1038/srep35162.

[157] C. Knipper, C. Meyer, F. Jacobi, C. Roth, M. Fecher, E. Stephan, et al., Social differentiation and land use at an Early Iron Age "princely seat": bioarchaeological investigations at the Glauberg (Germany), Journal of Archaeological Science. 41 (2014) 818-835. doi:10.1016/j.jas.2013.09.019.

[158] V.M. Oelze, J.K. Koch, K. Kupke, O. Nehlich, S. Zäuner, J. Wahl, et al., Multi-isotopic analysis reveals individual mobility and diet at the early iron age monumental tumulus of magdalenenberg, germany, American Journal of Physical Anthropology. 148 (2012) 406-421. doi:10.1002/ajpa.22063.

[159] R. Brettell, J. Evans, S. Marzinzik, A. Lamb, J. Montgomery, "Impious Easterners": Can Oxygen and Strontium Isotopes Serve as Indicators of Provenance in Early Medieval European Cemetery Populations?, European Journal of Archaeology. 15 (2012) 117-145. doi:10.1179/1461957112Y.0000000001.

[160] M. Jay, J. Montgomery, O. Nehlich, J. Towers, J. Evans, British Iron Age chariot burials of the Arras culture: a multi-isotope approach to investigating mobility levels and subsistence practices, World Archaeology. 45 (2013) 473-491. doi:10.1080/00438243.2013.820647.

[161] F.Ö. Dudás, S.A. LeBlanc, S.W. Carter, S.A. Bowring, Pb and Sr concentrations and isotopic compositions in prehistoric North American teeth: A methodological study, Chemical Geology. 429 (2016) 21-32. doi:10.1016/j.chemgeo.2016.03.003.

[162] M.A. Beherec, T.E. Levy, O. Tirosh, M. Najiar, K.A. Knabb, Y. Erel, Iron Age Nomads and their relation to copper smelting in Faynan (Jordan): Trace metal and $\mathrm{Pb}$ and $\mathrm{Sr}$ isotopic measurements from the Wadi Fidan 40 cemetery, Journal of Archaeological Science. 65 (2016) 70-83. doi:10.1016/j.jas.2015.10.006.

[163] S. di Lernia, M.A. Tafuri, Persistent deathplaces and mobile landmarks: The Holocene mortuary and isotopic record from Wadi Takarkori (SW Libya), Journal of Anthropological Archaeology. 32 
[164] T.D. Price, J.N. Nielsen, K.M. Frei, N. Lynnerup, Sebbersund: isotopes and mobility in an 11th-12th c. AD Danish churchyard, Journal of Archaeological Science. 39 (2012) 3714-3720. doi:10.1016/j.jas.2012.06.015

[165] V.M. OELZE, O. NEHLICH, M.P. RICHARDS, “THERE”S NO PLACE LIKE HOME'-NO ISOTOPIC EVIDENCE FOR MOBILITY AT THE EARLY BRONZE AGE CEMETERY OF SINGEN, GERMANY, Archaeometry. 54 (2012) 752-778. doi:10.1111/j.1475-4754.2011.00644.x.

1110

1111

1112

1113

1114

1115

1116

1117

1118

1119

1120

1121

1122

1123

1124

1125

1126

1127

1128

1129

1130

1131

1132

1133

1134

1135

1136

1137

1138

1139

1140

1141

1142

1143

1144

1145

1146

1147

1148

1149

1150

1151

1152

1153

1154

1155

1156

1157

1158

1159

1160

[166] S. Valenzuela-Lamas, S. Jiménez-Manchón, J. Evans, D. López, R. Jornet, U. Albarella, Analysis of seasonal mobility of sheep in Iron Age Catalonia (north-eastern Spain) based on strontium and oxygen isotope analysis from tooth enamel: First results, Journal of Archaeological Science: Reports. 6 (2016) 828-836. doi:10.1016/j.jasrep.2015.08.042.

[167] I. Scharlotta, A. Weber, Mobility of middle Holocene foragers in the Cis-Baikal region, Siberia: Individual life history approach, strontium ratios, rare earth and trace elements, Quaternary International. 348 (2014) 37-65. doi:10.1016/j.quaint.2014.03.040.

[168] M. Scheeres, C. Knipper, M. Hauschild, M. Schönfelder, W. Siebel, D. Vitali, et al., Evidence for "Celtic migrations"? Strontium isotope analysis at the early La Tène (LT B) cemeteries of Nebringen (Germany) and Monte Bibele (Italy), Journal of Archaeological Science. 40 (2013) 3614-3625. doi:10.1016/j.jas.2013.05.003.

[169] J.E. Laffoon, R. Rodríguez Ramos, L. Chanlatte Baik, Y. Narganes Storde, M. Rodríguez Lopez, G.R. Davies, et al., Long-distance exchange in the precolonial Circum-Caribbean: A multi-isotope study of animal tooth pendants from Puerto Rico, Journal of Anthropological Archaeology. 35 (2014) 220-233. doi:10.1016/j.jaa.2014.06.004.

[170] A.W. Weber, O.I. Goriunova, Hunter-gatherer migrations, mobility and social relations: A case study from the Early Bronze Age Baikal region, Siberia, Journal of Anthropological Archaeology. 32 (2013) 330-346. doi:10.1016/j.jaa.2012.01.006.

[171] T. Tütken, T.W. Vennemann, H.-U. Pfretzschner, Nd and Sr isotope compositions in modern and fossil bones - Proxies for vertebrate provenance and taphonomy, Geochimica et Cosmochimica Acta. 75 (2011) 5951-5970. doi:10.1016/j.gca.2011.07.024.

[172] M. Pérez de Nanclares, J.-E. Dessen, K.-A. Rørvik, Y. Thomassen, M.S. Thomassen, Feasibility of using rare earth elements (REEs) to mark and identify escaped farmed Atlantic salmon Salmo salar L., Aquaculture Research. 47 (2016) 1885-1898. doi:10.1111/are.12647.

[173] C. Lehn, A. Rossmann, M. Graw, Provenancing of unidentified corpses by stable isotope techniques - presentation of case studies., Science \& Justice : Journal of the Forensic Science Society. 55 (2015) 72-88. doi:10.1016/j.scijus.2014.10.006.

[174] C. Lehn, C. Lihl, A. Roßmann, Change of geographical location from Germany (Bavaria) to USA (Arizona) and its effect on $\mathrm{H}-\mathrm{C}-\mathrm{N}-\mathrm{S}$ stable isotopes in human hair, Isotopes in Environmental and Health Studies. 51 (2015) 68-79. doi:10.1080/10256016.2014.995645.

[175] S.J. McLean, H. Ikegaya, P.J. Saukko, H.Y. Zheng, T. Akutsu, D. Miyamori, et al., A trial of the utilization of stable isotope analysis for the estimation of the geographic origins of unidentified cadavers., Forensic Science International. 232 (2013) 237.e1-5. doi:10.1016/j.forsciint.2013.07.019.

[176] K. Alkass, H. Saitoh, B.A. Buchholz, S. Bernard, G. Holmlund, D.R. Senn, et al., Analysis of radiocarbon, stable isotopes and DNA in teeth to facilitate identification of unknown decedents, PloS One. 8 (2013) e69597. doi:10.1371/journal.pone.0069597.

[177] L. Font, G. van der Peijl, I. van Wetten, P. Vroon, B. van der Wagt, G. Davies, Strontium and lead isotope ratios in human hair: investigating a potential tool for determining recent human geographical movements, Journal of Analytical Atomic Spectrometry. 27 (2012) 719. doi:10.1039/c2ja10361c.

[178] M. Haeusler, C. Haas, S. Lösch, N. Moghaddam, I.M. Villa, S. Walsh, et al., Multidisciplinary Identification of the Controversial Freedom Fighter Jörg Jenatsch, Assassinated 1639 in Chur, Switzerland, PLOS ONE. 11 (2016) e0168014. doi:10.1371/journal.pone.0168014.

[179] L. Font, G. Jonker, P.A. van Aalderen, E.F. Schiltmans, G.R. Davies, Provenancing of unidentified World War II casualties: Application of strontium and oxygen isotope analysis in tooth enamel, Science \& Justice : Journal of the Forensic Science Society. 55 (2015) 10-7. doi:10.1016/j.scijus.2014.02.005.

[180] J.. Azparren, A. Fernandez-Rodriguez, G. Vallejo, Diagnosing death by drowning in fresh water using blood strontium as an indicator, Forensic Science International. 137 (2003) 55-59. doi:10.1016/S0379-0738(03)00284-6. 
[181] J.E. Azparren, C. Cubero, E. Perucha, P. Martínez, G. Vallejo, Comparison between lung weight and blood strontium in bodies found in seawater, Forensic Science International. 168 (2007) 128132. doi:10.1016/j.forsciint.2006.07.001.

[182] J.E. Azparren, E. Perucha, P. Martínez, R. Muñoz, G. Vallejo, Factors affecting strontium absorption in drownings, Forensic Science International. 168 (2007) 138-42. doi:10.1016/j.forsciint.2006.07.003.

[183] M.D. Pérez-Cárceles, S. del Pozo, A. Sibón, J.A. Noguera, E. Osuna, M.A. Vizcaya, et al., Serum biochemical markers in drowning: Diagnostic efficacy of Strontium and other trace elements, Forensic Science International. 214 (2012) 159-166. doi:10.1016/j.forsciint.2011.07.047.

[184] J.. Azparren, A. Ortega, H. Bueno, M. Andreu, Blood strontium concentration related to the length of the agonal period in seawater drowning cases, Forensic Science International. 108 (2000) 5160. doi:10.1016/S0379-0738(99)00200-5.

[185] M.D. Pérez-Cárceles, A. Sibón, M.L. Gil Del Castillo, M.A. Vizcaya, E. Osuna, T. Casas, et al., Strontium levels in different causes of death: diagnostic efficacy in drowning, Biological Trace Element Research. 126 (2008) 27-37. doi:10.1007/s12011-008-8180-1.

[186] J.C. Hegg, B.P. Kennedy, A.K. Fremier, Predicting strontium isotope variation and fish location with bedrock geology: Understanding the effects of geologic heterogeneity, Chemical Geology. 360-361 (2013) 89-98. doi:10.1016/j.chemgeo.2013.10.010.

[187] S.R. Brennan, C.E. Torgersen, J.P. Hollenbeck, D.P. Fernandez, C.K. Jensen, D.E. Schindler, Dendritic network models: Improving isoscapes and quantifying influence of landscape and instream processes on strontium isotopes in rivers, Geophysical Research Letters. 43 (2016) 5043 5051. doi:10.1002/2016GL068904.

[188] C.P. Bataille, G.J. Bowen, Mapping $87 \mathrm{Sr} / 86 \mathrm{Sr}$ variations in bedrock and water for large scale provenance studies, Chemical Geology. 304-305 (2012) 39-52. doi:10.1016/j.chemgeo.2012.01.028.

[189] C.P. Bataille, J. Laffoon, G.J. Bowen, Mapping multiple source effects on the strontium isotopic signatures of ecosystems from the circum-Caribbean region, Ecosphere. 3 (2012) art118. doi:10.1890/ES12-00155.1.

[190] L.M. Kootker, R.J. van Lanen, H. Kars, G.R. Davies, Strontium isoscapes in The Netherlands. Spatial variations in $87 \mathrm{Sr} / 86 \mathrm{Sr}$ as a proxy for palaeomobility, Journal of Archaeological Science: Reports. 6 (2016) 1-13. doi:10.1016/j.jasrep.2016.01.015.

[191] J.A. Evans, J. Montgomery, G. Wildman, N. Boulton, Spatial variations in biosphere 87Sr/86Sr in Britain, Journal of the Geological Society. 167 (2010) 1-4. doi:10.1144/0016-76492009-090.

[192] M. Willmes, L. McMorrow, L. Kinsley, R. Armstrong, M. Aubert, S. Eggins, et al., The IRHUM (Isotopic Reconstruction of Human Migration) database \&amp;ndash; bioavailable strontium isotope ratios for geochemical fingerprinting in France, Earth System Science Data. 6 (2014) 117122. doi:10.5194/essd-6-117-2014.

[193] N. Shalev, B. Lazar, L. Halicz, M. Stein, I. Gavrieli, A. Sandler, et al., Strontium Isotope Fractionation in Soils and Pedogenic Processes, Procedia Earth and Planetary Science. 7 (2013) 790-793. doi:10.1016/j.proeps.2013.03.074.

[194] L. Halicz, I. Segal, N. Fruchter, M. Stein, B. Lazar, Strontium stable isotopes fractionate in the soil environments?, Earth and Planetary Science Letters. 272 (2008) 406-411. doi:10.1016/j.epsl.2008.05.005

[195] J. Fietzke, A. Eisenhauer, Determination of temperature-dependent stable strontium isotope ( 88 $\mathrm{Sr} / 86 \mathrm{Sr}$ ) fractionation via bracketing standard MC-ICP-MS, Geochemistry, Geophysics, Geosystems. 7 (2006) n/a-n/a. doi:10.1029/2006GC001243.

[196] J. Ma, G. Wei, Y. Liu, Z. Ren, Y. Xu, Y. Yang, Precise measurement of stable ( $\delta 88 / 86 S r)$ and radiogenic (87Sr/86Sr) strontium isotope ratios in geological standard reference materials using MC-ICP-MS, Chinese Science Bulletin. 58 (2013) 3111-3118. doi:10.1007/s11434-013-5803-5.

[197] K.T. SMITH, G.W. WHITLEDGE, Evaluation of a stable-isotope labelling technique for mass marking fin rays of age-0 lake sturgeon, Fisheries Management and Ecology. 18 (2011) 168-175. doi:10.1111/j.1365-2400.2010.00771.x.

[198] L.A. Neymark, W.R. Premo, N.N. Mel'nikov, P. Emsbo, Precise determination of $\delta 88$ Sr in rocks, minerals, and waters by double-spike TIMS: a powerful tool in the study of geological, hydrological and biological processes, J. Anal. At. Spectrom. 29 (2014) 65-75. doi:10.1039/C3JA50310K.

[199] P.Z. Vroon, B. van der Wagt, J.M. Koornneef, G.R. Davies, Problems in obtaining precise and accurate $\mathrm{Sr}$ isotope analysis from geological materials using laser ablation MC-ICPMS, Analytical 
and Bioanalytical Chemistry. 390 (2008) 465-476. doi:10.1007/s00216-007-1742-9.

[200] J. Irrgeher, P. Galler, T. Prohaska, 87Sr/86Sr isotope ratio measurements by laser ablation multicollector inductively coupled plasma mass spectrometry: Reconsidering matrix interferences in bioapatites and biogenic carbonates, Spectrochimica Acta Part B: Atomic Spectroscopy. 125 (2016) 31-42. doi:10.1016/j.sab.2016.09.008.

[201] M. Willmes, L. Kinsley, M.-H. Moncel, R.A. Armstrong, M. Aubert, S. Eggins, et al., Improvement of laser ablation in situ micro-analysis to identify diagenetic alteration and measure strontium isotope ratios in fossil human teeth, Journal of Archaeological Science. 70 (2016) 102-116. doi:10.1016/j.jas.2016.04.017.

[202] C. B'Hymer, J.A. Caruso, Selenium speciation analysis using inductively coupled plasma-mass spectrometry, Journal of Chromatography A. 1114 (2006) 1-20. doi:10.1016/j.chroma.2006.02.063.

[203] O.F.X. Donard, F.M. Martin, Hyphenated techniques applied to environmental speciation studies, TrAC Trends in Analytical Chemistry. 11 (1992) 17-26. doi:10.1016/0165-9936(92)80115-M.

[204] J.A. Caruso, M. Montes-Bayon, Elemental speciation studies-new directions for trace metal analysis, Ecotoxicology and Environmental Safety. 56 (2003) 148-163. doi:10.1016/S01476513(03)00058-7.

[205] G. Fortunato, K. Mumic, S. Wunderli, L. Pillonel, J.O. Bosset, G. Gremaud, Application of strontium isotope abundance ratios measured by MC-ICP-MS for food authentication, Journal of Analytical Atomic Spectrometry. 19 (2004) 227. doi:10.1039/b307068a.

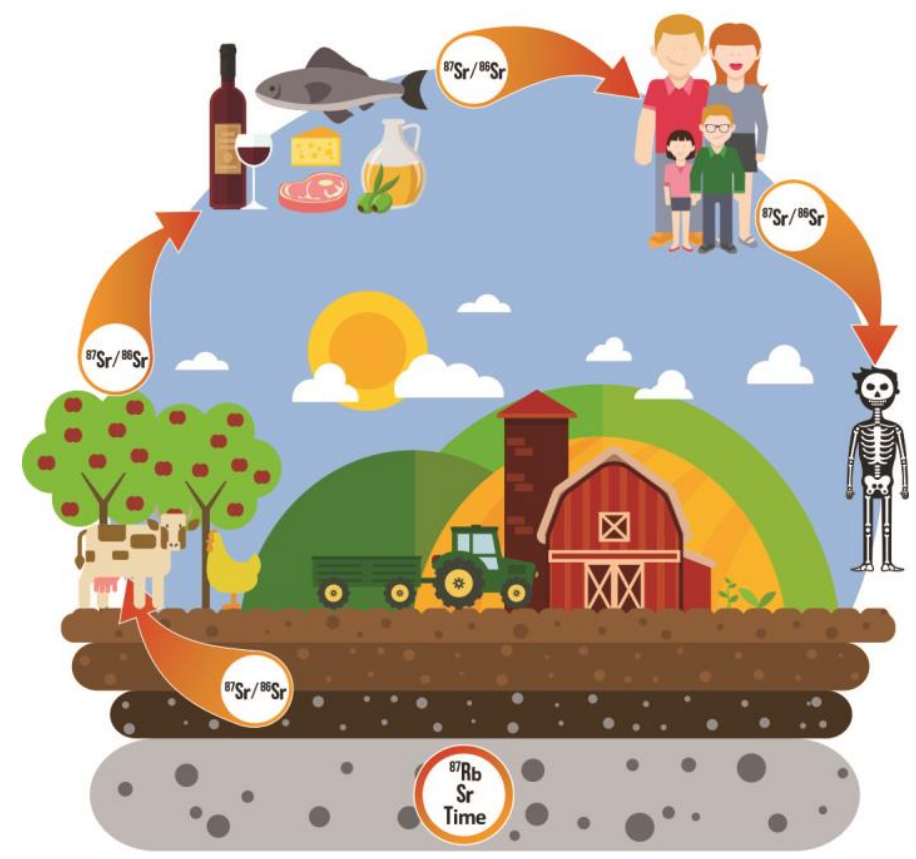

Fig.1. Conservative isotope ratio throughout the food chain 


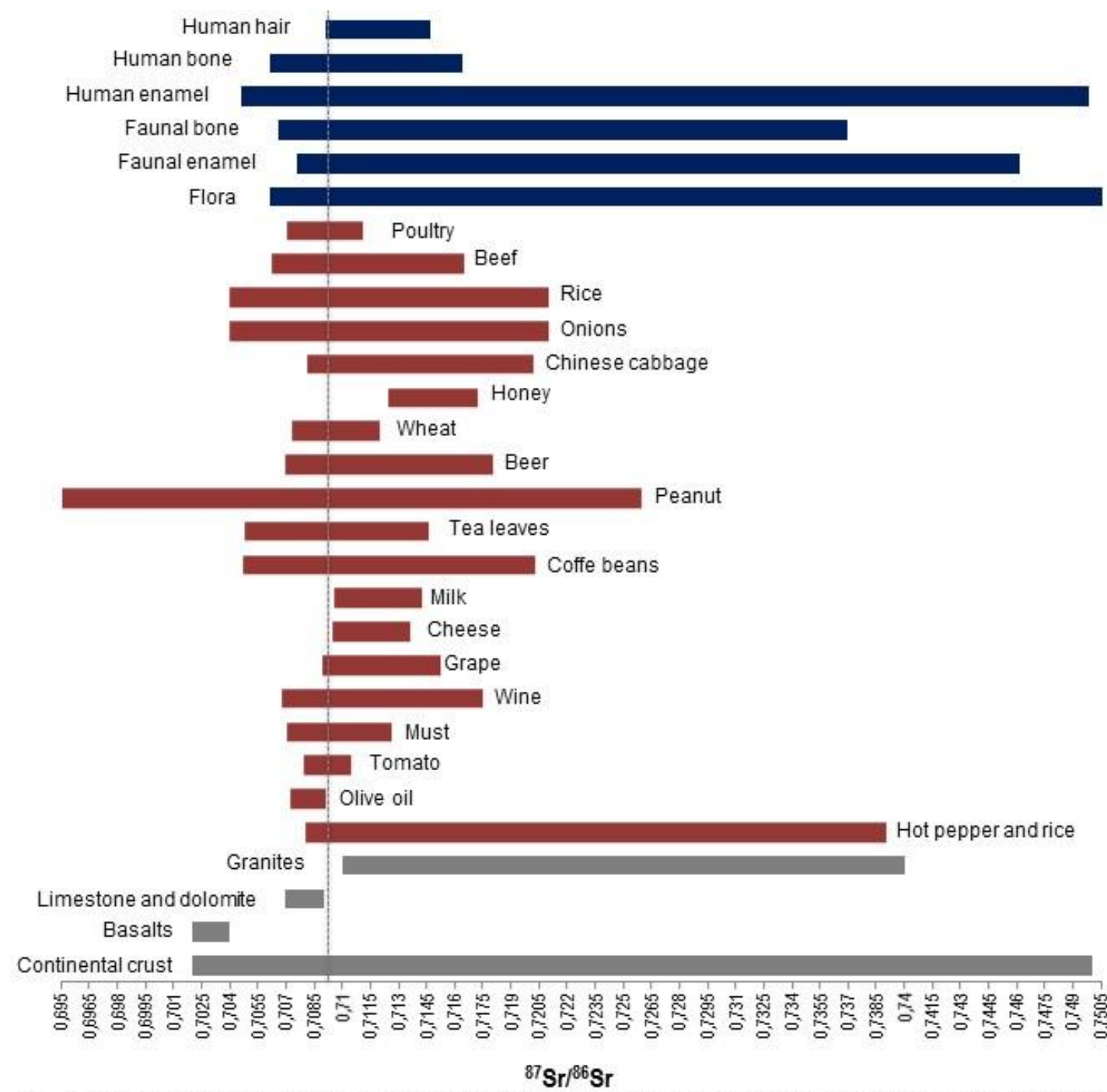

Fig 2 - Analysis of the ${ }^{87} \mathrm{Sr} /{ }^{85} \mathrm{Sr}$ per matrix. Blue bars represent samples from bioarchaeological studies, red bars represent foodstuffs and grey bars examples of the bedrock [6]. Vertical doted line represents modern seawater ratio (0.7092) [6] 